\title{
Charting the flavor landscape of MSSM-like Abelian heterotic orbifolds
}

\author{
Yessenia Olguín-Trejo, ${ }^{1, *}$ Ricardo Pérez-Martínez, ${ }^{1,2, \dagger}$ and Saúl Ramos-Sánchez,"\# \\ ${ }^{1}$ Instituto de Física, Universidad Nacional Autónoma de México, POB 20-364, Cd. Mx. 01000, México \\ ${ }^{2}$ Facultad de Ciencias Físico-Matemáticas, Universidad Autónoma de Coahuila, \\ Edificio A, Unidad Camporredondo, 25000, Saltillo, Coahuila, México
}

(Received 7 October 2018; published 26 November 2018)

\begin{abstract}
Discovering a selection principle and the origin of flavor symmetries from an ultraviolet completion of particle physics is an interesting open task. As a step in this direction, we classify all possible flavor symmetries of four-dimensional massless spectra emerging from supersymmetric Abelian orbifold compactifications, including roto-translations and nonfactorizable tori, for generic moduli values. Although these symmetries are valid in all string theories, we focus on the $E_{8} \times E_{8}$ heterotic string. We perform the widest known search of $\mathrm{E}_{8} \times \mathrm{E}_{8}$ Abelian orbifold compactifications, yielding over 94,000 models with MSSM-like features. About $70.5 \%$ of these models exhibit flavor symmetries containing $D_{4}$ factors and only nearly $1.5 \%$ have $\Delta(54)$ factors. The remaining models are furnished with purely Abelian flavor symmetries. Our findings suggest that should particle phenomenology arise from such a heterotic orbifold, it could accommodate only one of these flavor symmetries.
\end{abstract}

DOI: $10.1103 /$ PhysRevD.98.106020

\section{INTRODUCTION}

The reason for the number of families in the standard model (SM) as well as the origin of fermion mixings may be clarified in extensions of the SM. The general structure of the quark-mixing matrix motivated the bottom-up introduction of ad hoc discrete flavor symmetries (see e.g., $[1,2]$ for a review) that, together with a number of extra fields transforming in nontrivial flavor representations, yield new phenomenology that may be contrasted with observations. Choosing the correct flavor symmetry among the different scenarios that render similar physics requires a selection principle that is not found in this field-theoretic approach.

It is in this sense that, given the constraints of string theory and its potential to provide an ultraviolet completion of the SM, we can try to identify a mechanism in string theory to restrict the admissible flavor symmetries, providing thereby their origin. This quest is not new. The seminal works were in the context of heterotic orbifold compactifications $[3,4]$, which sparked the study of the phenomenological consequences of some models [5-9],

\footnotetext{
*yess.olt@ciencias.unam.mx

ricardoperezm@estudiantes.fisica.unam.mx

ramos@fisica.unam.mx
}

Published by the American Physical Society under the terms of the Creative Commons Attribution 4.0 International license. Further distribution of this work must maintain attribution to the author(s) and the published article's title, journal citation, and DOI. Funded by SCOAP . generalizations in models with magnetic fluxes [10] and relations with modular symmetries [11]. Flavor symmetries are associated in these works with geometric aspects of orbifolds, but they can also be related to larger continuous symmetries of the extra dimensions [12]. Also in D-brane compactifications, some sources of flavor symmetries have been identified and there is progress in the study of their phenomenology [13-16].

Here we focus on the $E_{8} \times E_{8}$ heterotic string compactified on all symmetric, toroidal, Abelian orbifolds ${ }^{1}$ that yield four-dimensional $\mathcal{N}=1$ low-energy effective field theories, recently classified in Ref. [22]. In these scenarios, the fact that most matter states are localized at the curvature singularities of the orbifold becomes instrumental to arrive at flavor phenomenology, because different singularities are assigned different localization numbers that can be interpreted as charges of a flavor symmetry in the fourdimensional resulting model.

In this work, we present first a systematic classification of flavor symmetries in Abelian toroidal orbifolds, whose moduli have no special values, avoiding possible enhancements. These symmetries are completely determined by the orbifold space group, whose nature is purely geometric, and are thus independent of the string theory to be compactified. As the geometric structure of a toroidal orbifold can be more complicated than usually assumed, due to the presence of roto-translations or nonfactorizable tori, this task can be challenging and lead to flavor

\footnotetext{
${ }^{1}$ Beside the original works $[17,18]$, there are several good introductions to these constructions, see e.g., [19-21].
} 
symmetries not yet identified. Since we explore here all sixdimensional orbifolds, this paper represents the completion of the work initiated in Ref. [23].

Orbifolds are used in the heterotic strings to obtain models that reproduce the main features of the SM [24], its minimal supersymmetric extension [25-28] (MSSM) and other nonminimal extensions [29], as well as many other observed and/or desirable properties of particle physics [30-36]. Aiming at gaining insight on the actual flavor symmetry of Nature, an interesting question we can pursue is: what flavor symmetries can these orbifolds have?

To answer this question, we perform a search of semirealistic $\mathcal{N}=1$ heterotic orbifolds with help of the orbifolder [37]. We then study their flavor symmetries, which build subgroups of the symmetries we classify in Sec. III. We expect that a statistical analysis of these findings may hint towards the family structure that particle physics emerging from strings can have.

This paper is organized as follows. After reviewing the aspects of heterotic orbifolds that are crucial for our study on flavor symmetries, we proceed in Sec. III to discuss how flavor symmetries arise in Abelian toroidal orbifolds. We then classify all flavor symmetries that can arise from these orbifolds, independently of the string theory one may compactify. In Sec. IV, we show the results of the most comprehensive search of semirealistic heterotic orbifolds so far. Section V is devoted to the discussion of the flavor symmetries that arise in the promising models we found, which are summarized in the tables presented in the Appendix. In Sec. VI, we provide our summary and outlook.

\section{ORBIFOLD COMPACTIFICATIONS}

\section{A. Toroidal orbifolds}

In order to introduce our notation and the main aspects of our constructions, let us first study the structure of six-dimensional toroidal orbifolds in the context of fourdimensional $\mathcal{N}=1$ models resulting from the supersymmetric heterotic strings.

In general, a six-dimensional toroidal orbifold $\mathcal{O}$ is defined as the quotient space that results from dividing a six-dimensional torus $\mathbb{T}^{6}$ by the so-called orbifolding group $G$. The torus can be embedded in $\mathbb{R}^{6}$ by dividing this space by a lattice $\Lambda$ with basis vectors $\left\{e_{i} \mid i=1, \ldots, 6\right\}$, corresponding to identifying all points of $\mathbb{R}^{6}$ connected by translations $\lambda \in \Lambda$, such that $\lambda=\sum_{i} m_{i} e_{i}$ for some integers $m_{i}$.

Alternatively, one can produce the same orbifold $\mathcal{O}$ by moding $\mathbb{R}^{6}$ by the space group $S$, which is a discrete group of isometries of the torus $\mathbb{T}^{6}$, including the translations in the lattice $\Lambda$. For our purposes, this description of an orbifold turns out to be more useful. That is, we shall consider here a six-dimensional toroidal orbifold defined as

$$
\mathcal{O}=\mathbb{R}^{6} / S
$$

The elements $g \in S$ have the general structure $g=(\vartheta, \mu)$, where the operators $\vartheta$ are in general elements of $\mathrm{O}(6)$ that form a discrete, Abelian or non-Abelian point group $P$ of $S$, and $\mu$ is a vector in $\mathbb{R}^{6}$, which may or may not be an element of the torus lattice, although it can always be written in the basis of $\Lambda$ (with arbitrary coefficients). The action of $g \in S$ on $x \in \mathbb{R}^{6}$ is defined by

$$
x \stackrel{g}{\mapsto} g x=\vartheta x+\mu,
$$

that is, $\vartheta$ denotes a rotation, reflection or inversion of $x$ whereas $\mu$ denotes a translation vector.

It is said that the action of $g$ is trivial on the torus only if it amounts to a lattice translation. This is because $\mathbb{T}^{6}=\mathbb{R}^{6} / \Lambda$; i.e., the torus is obtained by the identification $x \simeq x+\lambda, \lambda \in \Lambda$. It follows that, if $\mu$ is an element of the torus lattice, $\mu=\lambda \in \Lambda$, the only component of $g \in S$ that exerts a nontrivial action on the torus is $\vartheta$, since $\vartheta x$ and $\vartheta x+\lambda$ are identified on a toroidal orbifold.

When $\mu$ in Eq. (2) is chosen to be a more general vector, $\mu \notin \Lambda$, the space-group element $g=(\vartheta, \mu)$ is called a rototranslation. In this case, both $\vartheta$ and $\mu$ act nontrivially on the six-dimensional torus. One of the purposes of this work is to study this case with more attention, attempting to pave the path towards phenomenology of orbifolds with roto-translations.

In an orbifold, the space group defining the orbifold consists of a finite number of elements $g \in S$ called space group generators, their products, computed according to

$g^{\prime \prime}=g g^{\prime}=(\vartheta, \mu)\left(\vartheta^{\prime}, \mu^{\prime}\right)=\left(\vartheta \vartheta^{\prime}, \mu+\vartheta \mu^{\prime}\right), \quad g, g^{\prime}, g^{\prime \prime} \in S$,

and their conjugations. All elements of a space group $S$ can be grouped in different conjugacy classes $[g]=$ $\left\{h^{-1} g h, h \in S\right\}$. All elements of a conjugacy class are equivalent. Note that an element $g=\left(\vartheta, \mu+\sum_{i} m_{i} e_{i}\right)$, with $\mu \notin \Lambda$ or null, can be rewritten as $\prod_{i}\left(\mathbb{1}, e_{i}\right)^{m_{i}}(\vartheta, \mu)$. Therefore, the space group generators can be pure $\mathrm{O}(6)$ transformations, roto-translations or translations.

An additional property of orbifold generators is that each of them has an integer order $N$, such that $g^{N}$ is trivial on the torus, that is $g^{N}=(\mathbb{1}, \lambda)$ with $\lambda \in \Lambda$. We point out that this restricts the shape of the translation vectors $\mu \notin \Lambda$ of rototranslations $g=(\vartheta, \mu)$. The trivial action of $g^{N}$ on the torus implies that $\vartheta^{N}=\mathbb{1}$ and $\sum_{j=0}^{N-1} \vartheta^{j} \mu \in \Lambda$. Notice that, e.g., if $\vartheta \mu=\mu \neq 0$, then the translation vector is given as a fraction of lattice vector, $\mu=\frac{1}{N} \lambda$.

Let us focus now on Abelian orbifolds, which are the scope of this work. Complexifying the orbifold generators $g$, Eq. (2) becomes 


$$
z \stackrel{g}{\mapsto} g z=\vartheta z+\mu, \quad z, \mu \in \mathbb{C}^{3},
$$

with the complex coordinates of $z$ related by $z^{a}=x^{2 a-1}+$ $i x^{2 a}, a=1,2,3$, with the real coordinates $x \in \mathbb{R}^{6}$. In Abelian orbifolds, the complexified $\vartheta$ elements of the space group generators can be simultaneously diagonalized and represented as matrices of the form $\vartheta=\operatorname{diag}\left(e^{2 \pi \mathrm{i} v_{1}}\right.$, $\left.e^{2 \pi \mathrm{i} v_{2}}, e^{2 \pi \mathrm{i} v_{3}}\right)$, with $0 \leq\left|v_{a}\right|<1$. The vector $v=\left(v_{1}, v_{2}, v_{3}\right)$ is commonly called twist vector.

\section{Fixed points and roto-translations}

Space group generators $g$ with nontrivial twist $\vartheta$ have a nonfree action on $\mathbb{T}^{6}$. This implies that, in these cases, some points are left unaltered or fixed under $g$, which correspond to curvature singularities of the compact space. The simplest example of such a fixed point is $z=0$ for the space group element $g=(\vartheta, 0)$ with $\vartheta$ a rotation in six dimensions, but there are frequently more than one fixed points in these cases. The number and localization of the fixed points depend on the details of the torus (or, equivalently, the lattice $\Lambda$ ) and the space group element under consideration. There are as many inequivalent fixed points as conjugacy classes of $S$ with nontrivial twist.

Given a space group generator $g=(\vartheta, \mu)$, it follows from Eq. (4) that the associated fixed points $z_{f}$ satisfy the condition

$$
g z_{f}=\vartheta z_{f}+\mu=z_{f}+\lambda_{f}, \quad \lambda_{f} \in \Lambda,
$$

where the lattice translations are needed because the identity must happen in the torus. In order to obtain all inequivalent fixed points associated with $g$, one can take different choices of $\lambda_{f}$ and then select only those that are not related by space group elements. We note that, by using the product rule (3), defining $g_{f}=\left(\mathbb{1},-\lambda_{f}\right) g$ leads to the identity $g_{f} z_{f}=z_{f}$. The space group element $g_{f}$ is typically called the constructing element associated with the singularity $z_{f}$.

Let us illustrate the fixed point structure of an orbifold by using a $\mathbb{T}^{2} / \mathbb{Z}_{2} \times \mathbb{Z}_{2}$ orbifold with roto-translations. ${ }^{2}$ We define the orbifold through the space-group roto-translation generators $g_{1}=\left(\theta, \frac{1}{2} e_{1}\right)$ and $g_{2}=\left(\omega, \frac{1}{2} e_{2}\right)$, where $e_{1,2}$ are the orthogonal lattice generators of the torus and the $\mathrm{O}(6)$ generators are given by

$$
\theta=\left(\begin{array}{cc}
1 & 0 \\
0 & -1
\end{array}\right), \quad \omega=\left(\begin{array}{cc}
-1 & 0 \\
0 & 1
\end{array}\right)
$$

\footnotetext{
${ }^{2}$ In terms of the classification of Ref. [22], we refer here to a two-dimensional subsector of the nonlocal geometry $(2,5)$ of a six-dimensional $\mathbb{Z}_{2} \times \mathbb{Z}_{2}$ toroidal orbifold. By itself, the nonorientable geometry induced by this space group cannot be used to compactify a six-dimensional field theory as it cannot sustain chiral fermions. Belonging to a larger six-dimensional orbifold solves this issue. We thank H. P. Nilles for this observation.
}

satisfying $\theta^{2}=\omega^{2}=\mathbb{1}$, such that $g_{1}^{2}$ and $g_{2}^{2}$ have a trivial action on the torus, as expected for a $\mathbb{Z}_{2} \times \mathbb{Z}_{2}$ orbifold. Omitting the translational generators $\left(\mathbb{1}, e_{i}\right)$ and their conjugations, the space group comprises the conjugacy classes of the elements $\left\{g_{1}, g_{2}, g_{1} g_{2}, \mathbb{1}\right\}$.

Let us first focus on the element $g=g_{1} g_{2}=$ $\left(-\mathbb{1}, \frac{1}{2}\left(e_{1}-e_{2}\right)\right)$. By applying Eq. (5), we find four fixed points in the fundamental domain of the torus: $z_{f} \in\left\{\frac{1}{4}\left(e_{1}+e_{2}\right), \frac{1}{4}\left(e_{1}+3 e_{2}\right), \frac{1}{4}\left(3 e_{1}+e_{2}\right), \frac{3}{4}\left(e_{1}+e_{2}\right)\right\}$. One can easily verify that only two of these points are inequivalent; $\frac{1}{4}\left(e_{1}+e_{2}\right)$ is related to $\frac{3}{4}\left(e_{1}+e_{2}\right)$ and $\frac{1}{4}\left(e_{1}+3 e_{2}\right)$ is related to $\frac{1}{4}\left(3 e_{1}+e_{2}\right)$ in the torus by acting on them with $g_{1}$ or $g_{2}$. Thus, one can choose the fixed points $z_{f, 0}=\frac{1}{4}\left(e_{1}+e_{2}\right)$ and $z_{f, 1}=\frac{1}{4}\left(3 e_{1}+e_{2}\right)$ as the inequivalent fixed points associated with $g$. These points are depicted with bullets in Fig. 1. The constructing elements associated with the fixed points are given by $g_{f, 0}=$ $\left(-\mathbb{1}, \frac{1}{2}\left(e_{1}+e_{2}\right)\right)$ and $g_{f, 1}=\left(-\mathbb{1}, \frac{1}{2}\left(3 e_{1}+e_{2}\right)\right)$.

We consider now the element $g=g_{1}$. For this element, it turns out that Eq. (5) has no solution, revealing that there are no fixed points associated with this space group element. The same is true for $g_{2}$. This observation will be useful when figuring out the geometric discrete symmetries of the compactification.

For reasons that shall be clearer in Sec. II B, each set of fixed points is named a sector. From our previous discussion, we note that, ignoring the trivial sector of the identity element, in the $\mathbb{T}^{2} / \mathbb{Z}_{2} \times \mathbb{Z}_{2}$ orbifold worked out here there are two empty sectors and one sector with two fixed points. The appearance of empty sectors is related to the existence of roto-translation space group elements in toroidal Abelian orbifolds. In general, orbifolds without roto-translations do not exhibit empty sectors.

The global geometric structure of the orbifold is obtained by inspecting the action of all the space group generators. From the sector associated with $g=g_{1} g_{2}$, we find that the space group reduces the fundamental domain of $\mathbb{T}^{2}$ to $1 / 4$ of the torus fundamental domain, as illustrated in Fig. 1. We see that the combined action of $g_{1}$ and $g_{2}$ identifies the singularities depicted at the top with those in the bottom, sharing the symbols $\times$ and + . This crossed identification also affects the "boundaries" of the fundamental domain of the orbifold, which are also identified according to the types of arrows in the figure. From this description, we observe that this orbifold is equivalent to the well-known two-dimensional cross-capped pillow, with p-rectangular Bravais lattice (see e.g., Table B.2 of Ref. [22]), also called singular (real) projective plane.

The structure of fixed points in an orbifold allows to determine its geometric symmetries. Notice, in our example, that the cross-capped pillow is symmetric under the exchange of its inequivalent singularities. Consequently, this orbifold is invariant under $S_{2} \simeq \mathbb{Z}_{2}$ transformations. 

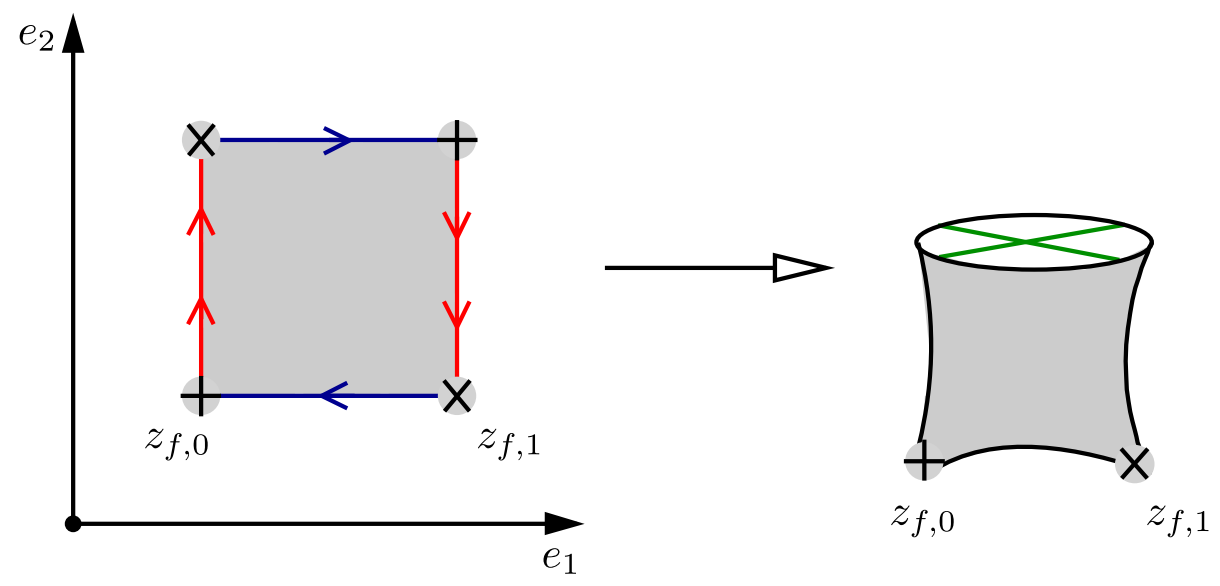

FIG. 1. A two-dimensional $\mathbb{Z}_{2} \times \mathbb{Z}_{2}$ orbifold with roto-translations. The shaded region on the left corresponds to the fundamental region of the orbifold. The fixed points $z_{f, 0}$ and $z_{f, 1}$ of the $g=g_{1} g_{2}$ sector are displayed with bullets in the figure. The fixed points at the top are identified with those in the bottom with the same symbol by the action of other space group elements. Similar arrows are also identified. This description is equivalent to the cross-capped pillow on the right.

Analogous (permutation) $S_{n}$ symmetries arise in orbifolds with a different number of singularities.

\section{B. Heterotic Abelian orbifolds}

The degrees of freedom (d.o.f.) of a string theory emerge from the left- and right-moving vibrational modes of a string. The observation that they are independent led to the heterotic strings, which are the mixture of the right-moving modes, $X_{R}$ and $\Psi_{R}$, of a ten-dimensional supersymmetric string with the left-moving modes, $X_{L}$ of a 26-dimensional bosonic string. The 16 extra bosonic d.o.f. $X_{L}^{I}, I=1, \ldots, 16$, are compactified on a torus $\mathbb{T}^{16}$, whose lattice vectors are constrained by anomaly cancellation to be those of the $\Lambda_{\mathrm{E}_{8} \times \mathrm{E}_{8}}$ or $\Lambda_{\mathrm{SO}(32)}$ root lattice, ${ }^{3}$ revealing the structure of an $\mathrm{E}_{8} \times \mathrm{E}_{8}$ or $\mathrm{SO}(32)$ gauge group on a tendimensional supersymmetric spacetime. We focus here on the heterotic string with $\mathrm{E}_{8} \times \mathrm{E}_{8}$ gauge group.

Heterotic orbifolds are constructed by compactifying six spatial dimensions of the ten-dimensional spacetime of a heterotic string on a toroidal orbifold. Right- and leftmoving modes, $X_{R}$ and $X_{L}$, mix to build the (bosonic) coordinates of the spacetime, $X=X_{L}+X_{R}$, but they can still be taken as independent d.o.f. As a consequence, one can in principle choose different compactification schemes for each mode. However, for simplicity, we focus here on so-called symmetric heterotic orbifolds, in which both modes are compactified on the same orbifold. As already mentioned, we can also complexify these coordinates, so that we have two uncompactified complex dimensions, corresponding to those of the observed spacetime, and three complex dimensions compactified on an Abelian orbifold.

\footnotetext{
${ }^{3}$ If one does not demand the resulting theory to be supersymmetric, there is a third option, the $\mathrm{SO}(16) \times \mathrm{SO}(16)$ root lattice.
}

Insisting on preserving $\mathcal{N}=1$ supersymmetry in four dimensions after compactifying the $\mathcal{N}=1$ heterotic strings on six-dimensional toroidal orbifolds, restricts a number of properties of these constructions. First, it is known that preserving $\mathcal{N}=1$ requires that the point group $P$ be a subgroup of SU(3). Recalling that the point group elements of Abelian orbifolds can be written as $\vartheta=\operatorname{diag}\left(e^{2 \pi \mathrm{i} v_{1}}\right.$, $\left.e^{2 \pi \mathrm{i} v_{2}}, e^{2 \pi \mathrm{i} v_{3}}\right)$, we immediately find that the condition $v_{1}+$ $v_{2}+v_{3}=0$ for each diagonalized generator leads to obtain $\mathcal{N}=1$ in four dimensions. Furthermore, more than two independent generators of $P$ would not leave any invariant supersymmetry generator; thus, only one or two distinct point group generators of orders $N$ and $M$ can be considered, corresponding to cyclic $\mathbb{Z}_{N}$ or $\mathbb{Z}_{N} \times \mathbb{Z}_{M}$ point groups. It is customary to label the orbifold by the name of its point group (also called $\mathbb{Q}$ class). In general, there is more than one (couple of) generator(s) that can yield the same point group, but any choice can be diagonalized in terms of the same twist vector.

Secondly, demanding that the space group elements be torus isometries further restricts both the choice of the tori and the space groups. For each choice of generators of a given point group, there are different torus lattices $\Lambda$ that are left invariant under the point group. If we allow for a number of moduli to take any values and consider the lattices so related to be equivalent, they build a so-called $\mathbb{Z}$ class. Each point group admits different $\mathbb{Z}$ classes.

Finally, once the point group and a torus lattice have been chosen for the compactification, one has still the freedom to consider different values of the translations $\mu$ of the space group generators $g=(\vartheta, \mu)$, which may be equivalent up to affine transformations or not. Equivalent translations together with the corresponding lattice and point group generators define an affine class.

In summary, all space groups useful for orbifold compactifications are obtained by classifying the admissible 
TABLE I. We list in the first column all 17 different Abelian point groups for six-dimensional toroidal heterotic orbifolds that yield $\mathcal{N}=1$ supersymmetric models in four dimensions. The second column displays the twist vectors associated with the point group generators. In the third and fourth columns, we show, respectively, the number of lattices (or $\mathbb{Z}$ classes) and space-group translations (or affine classes) that are compatible with each point group. The details of each space group are given in Ref. [22].

\begin{tabular}{|c|c|c|c|}
\hline Orbifold label & Twist vector(s) & $\#$ of $\mathbb{Z}$ classes & \# of affine classe \\
\hline $\mathbb{Z}_{3}$ & $\frac{1}{3}(1,1,-2)$ & 1 & 1 \\
\hline $\mathbb{Z}_{4}$ & $\frac{1}{4}(1,1,-2)$ & 3 & 3 \\
\hline $\mathbb{Z}_{6}-\mathrm{I}$ & $\frac{1}{6}(1,1,-2)$ & 2 & 2 \\
\hline $\mathbb{Z}_{6}-\mathrm{II}$ & $\frac{1}{6}(1,2,-3)$ & 4 & 4 \\
\hline $\mathbb{Z}_{7}$ & $\frac{1}{7}(1,2,-3)$ & 1 & 1 \\
\hline $\mathbb{Z}_{8}-\mathrm{I}$ & $\frac{1}{8}(1,2,-3)$ & 3 & 3 \\
\hline $\mathbb{Z}_{8}-\mathrm{II}$ & $\frac{1}{8}(1,3,-4)$ & 2 & 2 \\
\hline $\mathbb{Z}_{12}-\mathrm{I}$ & $\frac{1}{12}(1,4,-5)$ & 2 & 2 \\
\hline $\mathbb{Z}_{12}-\mathrm{II}$ & $\frac{1}{12}(1,5,-6)$ & 1 & 1 \\
\hline $\mathbb{Z}_{2} \times \mathbb{Z}_{2}$ & $\frac{1}{2}(0,1,-1), \frac{1}{2}(1,0,-1)$ & 12 & 35 \\
\hline $\mathbb{Z}_{2} \times \mathbb{Z}_{4}$ & $\frac{1}{2}(0,1,-1), \frac{1}{4}(1,0,-1)$ & 10 & 41 \\
\hline $\mathbb{Z}_{2} \times \mathbb{Z}_{6}-\mathrm{I}$ & $\frac{1}{2}(0,1,-1), \frac{1}{6}(1,0,-1)$ & 2 & 4 \\
\hline $\mathbb{Z}_{2} \times \mathbb{Z}_{6}-\mathrm{II}$ & $\frac{1}{2}(0,1,-1), \frac{1}{6}(1,1,-2)$ & 4 & 4 \\
\hline $\mathbb{Z}_{3} \times \mathbb{Z}_{3}$ & $\frac{1}{3}(0,1,-1), \frac{1}{3}(1,0,-1)$ & 5 & 15 \\
\hline $\mathbb{Z}_{3} \times \mathbb{Z}_{6}$ & $\frac{1}{3}(0,1,-1), \frac{1}{6}(1,0,-1)$ & 2 & 4 \\
\hline $\mathbb{Z}_{4} \times \mathbb{Z}_{4}$ & $\frac{1}{4}(0,1,-1), \frac{1}{4}(1,0,-1)$ & 5 & 15 \\
\hline $\mathbb{Z}_{6} \times \mathbb{Z}_{6}$ & $\frac{1}{6}(0,1,-1), \frac{1}{6}(1,0,-1)$ & 1 & 1 \\
\hline
\end{tabular}

combinations of point groups and their $\mathbb{Z}$ and affine classes. This has been done systematically in Ref. [22], from which we learn that there exist 138 admissible Abelian space groups for six-dimensional supersymmetric orbifold. All possible point groups with their corresponding twist vectors and the number of compatible $\mathbb{Z}$ and affine classes are listed in Table I. We shall explore the phenomenology of all 138 space groups.

Once one space group has been chosen to compactify the heterotic strings, the geometric features of the orbifold in six dimensions are completely defined, and, due to the conformal structure of string theory, these properties determine some aspects of the spectrum of matter in the resulting four-dimensonal supersymmetric field theory. In particular, modular invariance of the heterotic string requires that the orbifold action be embedded into the gauge d.o.f. of the string. This means that the space group must be translated into an equivalent group acting in the 16-dimensional space associated with the gauge group, the so-called gauge twisting group.

The simplest such an embedding is defined (in the bosonic formulation) by two kinds of translations of the gauge d.o.f. The point group elements $\vartheta$ are embedded as shifts $V$, whereas the torus lattice vectors $e_{i}$ are embedded as so-called Wilson lines (WLs) $A_{i}, i=1, \ldots, 6$. Let us explain the details by using $\mathbb{Z}_{N} \times \mathbb{Z}_{M}$ orbifolds as our working example. A generic space group element $g$ of a $\mathbb{Z}_{N} \times \mathbb{Z}_{M}$ orbifold with $P$ generators $\theta$ and $\omega$ can be embedded into the gauge d.o.f. as

$$
g=\left(\theta^{n} \omega^{m}, \mu_{i} e_{i}\right) \hookrightarrow V_{g} \equiv n V+m W+\mu_{i} A_{i},
$$

$n, m \in \mathbb{Z}, \mu_{i} \in \mathbb{R}$,

where $V$ and $W$ are the 16-dimensional shift vectors of fractional entries that encode in the gauge group the respective action of $\theta$ and $\omega$ in the six-dimensional orbifold; $\mu_{i}$ are nonintegers or integer numbers, depending on whether the space group element is a roto-translation or not; and the six WLs $A_{i}$ are also 16-dimensional fractional vectors. $V_{g}$ represents the gauge embedding of the space group $g$.

Under this gauge embedding, the action of a space group element is such that $z \mapsto g z$ in six of the ten dimensions of the spacetime of the heterotic string, and the bosonic leftmoving coordinates associated with the gauge d.o.f. of the heterotic strings are transformed according to

$$
X_{L}^{I} \mapsto X_{L}^{I}+2 \pi V_{g}^{I}, \quad I=1, \ldots, 16 .
$$

It is convenient to discuss the details of the states associated with the string excitations in the dual momentum space. If we focus on the gauge momentum contribution to the states $|p\rangle_{L}$ with momentum $p$, its behavior under the action of a space group element is dominated by the leftmoving contribution to the full vertex operator $\exp \{\mathrm{i} p$. $\left.X_{L}\right\}$ (see e.g., Eq. (2.5) of Ref. [38]). Under the action of $g$, this operator becomes $\exp \left\{2 \pi \mathrm{i} p \cdot V_{g}\right\} \exp \left\{\mathrm{i} p \cdot X_{L}\right\}$, which means that the momentum state acquires a phase under $g$, 


$$
|p\rangle_{L} \rightarrow e^{2 \pi \mathrm{i} p \cdot V_{g}}|p\rangle_{L}, \quad p \in \Lambda_{\mathrm{E}_{8} \times \mathrm{E}_{8}},
$$

where $\Lambda_{\mathrm{E}_{8} \times \mathrm{E}_{8}}$ denotes the (self-dual and integer) root lattice of the $\mathrm{E}_{8} \times \mathrm{E}_{8}$ gauge group.

The gauge embedding is subject to some constraints. First, since the point group generators of a $\mathbb{Z}_{N} \times \mathbb{Z}_{M}$ orbifold satisfy $\theta^{N}=\mathbb{1}=\omega^{M}$, the action of the shift vectors corresponding to $\theta^{N}$ and $\omega^{M}$ must be trivial in the gauge d.o.f. This implies that, according to Eq. (9), the shift vectors are constrained to satisfy $N V, M W \in \Lambda_{\mathrm{E}_{8} \times \mathrm{E}_{8}}$ because the lattice is integer (i.e., the inner product of different lattice vectors is an integer). Secondly, WLs must be consistent with the torus geometry and the orbifold action on it. For a given point group generator, in general, $\vartheta e_{i}=\sum_{j} \gamma_{i j} e_{j}$ for some integer coefficients $\gamma_{i j}$. This implies that the WLs must fulfill the relations $A_{i}=\gamma_{i j} A_{j}$ up to lattice translations in $\Lambda_{\mathrm{E}_{8} \times \mathrm{E}_{8}}$. The set of resulting equations of this type can be reduced to conditions for the WLs; some of them must vanish and other WLs $A_{i}$ have a nontrivial order $N_{i}$, such that $N_{i} A_{i} \in \Lambda_{\mathrm{E}_{8} \times \mathrm{E}_{8}}$ (without summation over $i$ ).

The final constraint on the gauge embedding comes from modular invariance, which is a string theoretical requirement ensuring that the compactified field theory is anomaly free. In the most general case of Abelian $\mathbb{Z}_{N} \times \mathbb{Z}_{M}$ heterotic orbifolds, modular invariance requires that [39]

$$
\begin{array}{rlll}
N\left(V^{2}-v^{2}\right)=0 & \bmod 2, & N_{i}\left(V \cdot A_{i}\right)=0 \bmod 2, \quad i=1, \ldots, 6, \\
M\left(W^{2}-w^{2}\right)=0 & \bmod 2, & N_{i}\left(W \cdot A_{i}\right)=0 \bmod 2, & \\
M(V \cdot W-v \cdot w)=0 & \bmod 2, & N_{i} A_{i}^{2}=0 \bmod 2, \\
& & \operatorname{gcd}\left(N_{i}, N_{j}\right)\left(A_{i} \cdot A_{j}\right)=0 \bmod 2, \quad i \neq j .
\end{array}
$$

Here we consider $\theta=\operatorname{diag}\left(e^{2 \pi \mathrm{i} v_{1}}, e^{2 \pi \mathrm{i} v_{2}}, e^{2 \pi \mathrm{i} v_{3}}\right)$ and $\omega=$ $\operatorname{diag}\left(e^{2 \pi \mathrm{i} w_{1}}, e^{2 \pi \mathrm{i} w_{2}}, e^{2 \pi \mathrm{i} w_{3}}\right)$ in terms of the two twist vectors, $v$ and $w$.

The space group together with the corresponding gauge twisting group, fulfilling all the previous requirements, builds up an admissible symmetric, Abelian orbifold compactification of a heterotic string.

The properties of the space group and a compatible gauge twisting group completely determine the matter content of the emerging four-dimensional field theory. The matter fields in a heterotic orbifold correspond to the quantum states of (left- and right-moving) closed string modes, that are left invariant under the action of all elements of the space and gauge twisting groups. String modes that are not invariant under the orbifold do not build admissible states of the compactification. Closed strings in an orbifold are of two kinds: untwisted and twisted strings. Untwisted strings are closed strings found among the original strings of the ten-dimensional heterotic theory and that are not projected out by the orbifold action. Twisted strings are special. They arise only because of the appearance of the orbifold singularities and are thus attached to them.

As in the uncompactified theory, four-dimensional effective states consist of a left- and a right-moving component. Both components must fulfill the so-called levelmatching condition, $M_{R}=M_{L}$, whose origin is that there is no preferred point on a closed string. For nonzero masses of string states are few times the string scale $M_{s}$, which is close to the Planck scale, any massive state is too massive to be observed at low energies and, therefore, decouples from the observable matter spectrum of the compactification.
In string compactifications aiming at reproducing the physics of our universe, one must thus focus on the study of massless (super)fields, $M_{L}=M_{R}=0$.

Since in ten dimensions the only massless closed strings found in the heterotic theory are those corresponding to the $\mathrm{E}_{8} \times \mathrm{E}_{8}$ superfields and the gravity supermultiplet, the untwisted closed-string states that are invariant under the orbifold represent first the unbroken four-dimensional gauge superfields that generate the unbroken gauge group $\mathcal{G}_{4 D} \subset \mathrm{E}_{8} \times \mathrm{E}_{8}$, and the four-dimensional gravity multiplet. Additionally, they correspond to the (untwisted) moduli, which parametrize the size and shape of the orbifold, and some (untwisted) matter fields that transform nontrivially under $\mathcal{G}_{4 D}$. The gauge properties of heterotic string fields are determined by their left-moving momentum, which for untwisted fields is just a vector of the root lattice of the tendimensional gauge group, $p \in \Lambda_{\mathrm{E}_{8} \times \mathrm{E}_{8}}$. Those states whose momenta satisfy $p \cdot V=p \cdot W=p \cdot A_{i}=0 \bmod 1$ belong to the gravity multiplet, the gauge multiplets or are moduli; the rest of the states have nontrivial gauge quantum numbers and build therefore matter fields.

The twisted states correspond to closed strings whose center of mass is at the orbifold singularities. Their left- and right-moving momenta depend on the constructing element associated with the singularity to which they are attached, according to our discussion in Sec. II A 1. The matter spectrum of string states of an orbifold is mostly populated by twisted fields. The gauge momentum of a string attached to the fixed point associated with the constructing element $g$ is given by $p_{s h}=p+V_{g}$, where $V_{g}$ is defined in Eq. (7). The corresponding states remain in the orbifold spectrum only if they are invariant under the action of all centralizer 
elements $h \in S$, such that $[g, h]=0$. It is thus clear that, when some WL is chosen to vanish, $A_{j}=0$ for some fixed $j$ (up to lattice translations), four-dimensional matter fields located at the singularities with constructing elements $\left(\theta^{n} \omega^{m}, \mu_{j} e_{j}+\sum_{i \neq j} \mu_{i} e_{i}\right)$ and $\left(\theta^{n} \omega^{m}, \mu_{j}^{\prime} e_{j}+\sum_{i \neq j} \mu_{i} e_{i}\right)$ are identical concerning their quantum numbers under $\mathcal{G}_{4 D}$, as long as their centralizers are equivalent. Following the final remarks in Sec. II A 1, those states would nevertheless be related under the internal geometric (permutation) symmetry of the orbifold. However, if $A_{j} \neq 0, p_{s h}$ at various singularities differ, breaking the permutation symmetry. These are key observations to arrive at the flavor symmetries, as we now proceed to discuss.

\section{FLAVOR SYMMETRIES IN ABELIAN HETEROTIC ORBIFOLDS}

\section{A. Symmetries from string selection rules}

As long as the strings are not deformed by the spacetime curvature, conformal field theory (CFT) is a useful tool to compute, e.g., the amplitude of the interactions among the fields related to the string states [40-43]. Since orbifolds are flat everywhere but at isolated points, the description of the string dynamics is just as in the original uncompactified theory, even after compactification on these spaces. This is a great advantage of orbifold compactifications because we must not rely on a supergravity approximation, which might break the connection between string theory and the fourdimensional effective model.

In the CFT, one determines the coupling strength of interactions among, say, $r$ effective fields $\Phi_{\ell}, \ell=1, \ldots, r$, by computing the $r$-point correlation functions of the vertex operators associated with the interacting fields,

$$
\mathcal{A}=\left\langle V_{-1 / 2}^{(1)} V_{-1 / 2}^{(2)} V_{-1}^{(3)} V_{0}^{(4)} \cdots V_{0}^{(r)}\right\rangle,
$$

where $V_{-1 / 2}^{(\ell)}$ denotes a fermionic vertex operator in the $(-1 / 2)$-ghost picture and $V_{0,-1}^{(\ell)}$ denote bosonic vertex operators in the 0 or $(-1)$-ghost pictures. The explicit expressions are written in terms of the quantum numbers of the string states (cf. e.g., [38]), revealing that there is a number of conditions that those quantum numbers must satisfy in order for the interaction amplitudes (11) to be nonvanishing. These conditions are known as selection rules [38,44-49]. The selection rules, beside gauge invariance, include $R$-charge conservation and space-group invariance, which deserve a discussion because they lead to discrete symmetries that may be important for flavor physics.

\section{R-charge conservation}

In addition to the left-moving momentum $p_{s h}$ that contains the information about its gauge charges, a string state has the so-called $H$-momentum $q_{s h}$ in the three compactified, complex dimensions $z^{a}$. In the bosonic formulation of the heterotic string, the entries of the $H$-momentum are fractional numbers that depend on whether they correspond to the description of a fermion or a boson, differing by $\pm 1 / 2$ units. This momentum, together with the number of left- and right-moving oscillator perturbations acting on the vacuum, build the socalled $R$-charge (see e.g., $[48,49]$ ), which, in contrast to pure $H$-momentum, is invariant under the ghost picturechanging operation. ${ }^{4}$

By computing CFT correlation functions (11), one can demonstrate that weakly-coupled strings interact only if the total $R$-charge of the coupling satisfies a conservation principle stated as $[48,49]$

$$
\sum_{\ell=1}^{r} R_{a}^{(\ell)}=-1 \quad \bmod N_{a}, \quad a=1,2,3,
$$

where each integer $N_{a}$ denotes the order of the point group generators acting on the ath complex coordinate $z^{a}$ of the six-dimensional torus, i.e., such that $N_{a} v_{a} \in \mathbb{Z}$. If one normalizes the charges $R_{a}^{(\ell)}$ to be integers by multiplying by $N_{a}$, Eq. (12) provides the discrete symmetry group $\mathbb{Z}_{N_{1}^{2}} \times \mathbb{Z}_{N_{2}^{2}} \times \mathbb{Z}_{N_{3}^{2}}$.

On the other hand, since these $R$-charges distinguish the bosonic and fermionic components of the four-dimensional effective superfields, the discrete symmetry arising from this invariance principle can be only an $R$ symmetry, explaining why they are called $R$-charges. We assume here that flavor symmetries are not $R$ symmetries, thus the discrete, $\mathbb{Z}_{N_{1}^{2}} \times \mathbb{Z}_{N_{2}^{2}} \times \mathbb{Z}_{N_{3}^{2}}$ symmetry of $R$-charges cannot be part of a flavor symmetry.

\section{Space-group invariance}

In the compactified theory, interactions must be invariant under the space group that defines the orbifold compactification. This implies that the joint action of the composition of the constructing elements of the interacting strings must be trivial on the orbifold (rather than on the torus). This condition is the so-called spacegroup selection rule. If we denote the constructing element of the fixed point $z_{f, \ell}$ of the sector $\left(\vartheta_{\ell}, \mu^{(\ell)}\right)$ as $g_{f}^{(\ell)}=\left(\vartheta_{\ell}, \mu_{f}^{(\ell)}\right)$, the space-group selection rule is given by

\footnotetext{
${ }^{4}$ The ghost picture or ghost charge of the vertex operators is given as subindex in Eq. (11). The total ghost charge must be -2 to cancel the ghost charge +2 of the sphere on which $\mathcal{A}$ is computed. However, all different ghost-charge assignations or pictures yielding the same total ghost charge provide equivalent results. Thus, it is natural to demand that physical charges be invariant under ghost-picture changing.
} 


$$
\prod_{\ell=1}^{r} g_{f}^{(\ell)}=\prod_{\ell=1}^{r}\left(\vartheta_{\ell}, \mu_{f}^{(\ell)}\right) \stackrel{!}{=}\left(\mathbb{1}, \bigcup_{\ell} \tilde{\Lambda}_{\ell}\right), \quad \tilde{\Lambda}_{\ell}=\left(\mathbb{1}-\vartheta_{\ell}\right) \Lambda,
$$

where e.g., $\vartheta_{\ell}=\theta^{q_{\ell}} \omega^{w_{\ell}}$ for $\mathbb{Z}_{N} \times \mathbb{Z}_{M}$ orbifolds and $\tilde{\Lambda}_{\ell}$ denotes the invariant sublattice of fixed points. The invariant sublattice of fixed points is such that, if the fixed point $z_{f, \ell}$ has constructing element $g_{f}^{(\ell)}$ and $\tilde{\lambda}^{(\ell)}=$ $\left(\mathbb{1}-\vartheta_{\ell}\right) \lambda$ with arbitrary $\lambda \in \Lambda$, then $z_{f, \ell}+\lambda$ is the fixed point associated with the constructing element $\left(\vartheta_{\ell}, \mu_{f}^{(\ell)}+\right.$ $\left.\tilde{\lambda}^{(\ell)}\right)$ which is in the conjugacy class of $g_{f}^{(\ell)}$ and refers thus to the same fixed point in the orbifold.

In order to satisfy the space-group selection rule, we must impose first that $\prod_{\ell} \vartheta_{\ell} \stackrel{!}{=} \mathbb{1}$, which for $\mathbb{Z}_{N} \times \mathbb{Z}_{M}$ orbifolds amounts to demanding

$$
\sum_{\ell=1}^{r} q_{\ell} \stackrel{!}{=} 0 \quad \bmod N, \quad \sum_{\ell=1}^{r} w_{\ell} \stackrel{!}{=} 0 \quad \bmod M .
$$

These relations suggest that the effective fields $\Phi_{\ell}$ can be considered to transform under a discrete symmetry $\mathbb{Z}_{N} \times \mathbb{Z}_{M}$ with charges $\left(q_{\ell}, w_{\ell}\right)$. Nonetheless, as we shall shortly see, these two symmetries are not always independent, yielding sometimes a smaller symmetry.

The second part of the space-group selection rule can be rewritten as

$$
\mu_{f}^{(1)}+\sum_{\ell=2}^{r}\left(\prod_{\ell^{\prime}=1}^{\ell-1} \vartheta_{\ell^{\prime}}\right) \mu_{f}^{(\ell)} \stackrel{!}{=} \sum_{\ell=1}^{r} \tilde{\lambda}^{(\ell)}, \quad \tilde{\lambda}^{(\ell)} \in \tilde{\Lambda}_{\ell}
$$

Since all vectors $\tilde{\lambda}^{(\ell)}$ and $\mu_{f}^{(\ell)}$ can be expressed in terms of the basis vectors $e_{i}, i=1, \ldots, 6$, Eq. (15) becomes a set of (up to) six independent conditions similar to those of Eq. (14), which depend on the specifics of the space group elements; i.e., the four-dimensional fields are charged under additional $\mathbb{Z}_{N_{i}}, i=1, \ldots, 6$, that depend on the space group.

To illustrate the conditions that follow from Eq. (15), let us consider the $\mathbb{T}^{2} / \mathbb{Z}_{2} \times \mathbb{Z}_{2}$ orbifold with the point-group generators given by Eq. (6), ignoring the rest of the sixdimensional heterotic orbifold (see footnote 2). A generic element $\lambda \in \Lambda$ is written as $\lambda=\lambda_{1} e_{1}+\lambda_{2} e_{2}$ with $\lambda_{i} \in \mathbb{Z}$. Let us suppose that we are considering couplings among states arising only from the sector $(\theta \omega, \mu)=\left(-1, \frac{1}{2}\left(e_{1}-\right.\right.$ $\left.e_{2}\right)$ ) since no fixed points appear in the $g_{1}$ and $g_{2}$ sectors of this orbifold. Because of Eq. (14) and $q_{\ell}=w_{\ell}=1$ for all massless twisted states we consider, we learn that the number $r$ of fields that an admissible coupling can have in this orbifold is even. A general element of the corresponding invariant sublattice is given by $(\mathbb{1}-\vartheta) \lambda=2 \lambda=$ $2 \lambda_{1} e_{1}+2 \lambda_{2} e_{2}$. Thus, we see that Eq. (15) takes the form

$$
\mu^{(1)}-\mu^{(2)}+\mu^{(3)}-\mu^{(4)}+\cdots-\mu^{(r)} \stackrel{!}{=} 2 \lambda,
$$

where the sign in the last vector is a consequence of $r$ being even. Rewriting the constructing elements as $g_{f, 0}=$ $\left(-\mathbb{1}, \mu+e_{2}\right)$ and $g_{f, 1}=\left(-\mathbb{1}, \mu+e_{1}+e_{2}\right)$, so that the field $\Phi_{\ell}$ in a coupling may have the constructing element $g_{f}^{(\ell)}=\left(-\mathbb{1}, \mu+n_{1}^{(\ell)} e_{1}+n_{2}^{(\ell)} e_{2}\right)$ with $\left(n_{1}^{(\ell)}, n_{2}^{(\ell)}\right)=(0,1)$ or $(1,1)$, we find that Eq. (16) yields two (apparently) independent conditions

$n_{1}^{(1)}+n_{1}^{(2)}+n_{1}^{(3)}+n_{1}^{(4)}+\cdots+n_{1}^{(r)} \stackrel{!}{=} 0 \bmod 2$,

$n_{2}^{(1)}+n_{2}^{(2)}+n_{2}^{(3)}+n_{2}^{(4)}+\cdots+n_{2}^{(r)} \stackrel{!}{=} 0 \bmod 2$,

where we have used that the integer $\lambda_{i}$ can be replaced by $\lambda_{i}^{\prime}-n_{i}^{(2)}-n_{i}^{(4)}-\cdots-n_{i}^{(r)}, i=1,2$, without loss of generality. Another important observation is that the orbifold sectors corresponding to the generators $g_{1}$ and $g_{2}$ do not lead to fixed points. This implies that there are no massless twisted states related to those sectors. Thus, if one focuses on massless twisted states, our previous considerations are enough to arrive at the flavor symmetry in the effective theory.

From our discussion, one could be tempted to conclude that the discrete symmetry emerging from the space group is $\mathbb{Z}_{2}^{4}$. This is wrong. The correct discrete symmetry that massless states support is only a $\mathbb{Z}_{2} \times \mathbb{Z}_{2}$. The reason is as follows. First, since the point-group charges of these states satisfy $q_{\ell}=w_{\ell}=1$, if $\sum_{\ell} q_{\ell}=r=0 \bmod 2$, then $\sum_{\ell} w_{\ell}=r=0 \bmod 2$ too. That is, we obtain only one independent $\mathbb{Z}_{2}$ from these conditions. Similarly, the second equation of (17) is automatically fulfilled once $r=0 \bmod 2$ has been imposed because we have chosen $\left(n_{1}^{(\ell)}, n_{2}^{(\ell)}\right)=(0,1)$ or $(1,1)$. However, there exists a nontrivial condition yielding a $\mathbb{Z}_{2}$ that does contribute to the flavor symmetry of massless states in the sample $\mathbb{T}^{2} / \mathbb{Z}_{2} \times \mathbb{Z}_{2}$ orbifold that we study here. ${ }^{5}$

It must be stressed that the symmetries that we have discussed are only related to massless states. Massive string states can wind on a torus even if it has no fixed points. In our example, this case would correspond to constructing elements such as $g_{1}$ or $g_{2}$. When all elements of the space group are taken into account, the corresponding symmetry becomes larger and the charges associated with the pointgroup generators and translations combine. Nevertheless, in this paper we shall only consider massless states and leave the general discussion for future work [50].

\section{B. General structure of flavor symmetries}

In orbifold compactifications (of any string theory), flavor symmetries can arise from the properties of the

\footnotetext{
${ }^{5}$ It is possible to show that $n_{1}^{(\ell)}+n_{2}^{(\ell)}$ is the only $\mathbb{Z}_{2}$ charge that is independent of the choice of the constructing elements we take (from their conjugacy classes). We thank Patrick K. S. Vaudrevange for very useful discussions and insight on this topic.
} 
space group. In particular, in heterotic orbifolds, they emerge as a result of combining the geometric properties of the extra dimensions and the symmetries emerging from the selection rules that we examined in the previous section.

As we have illustrated in Sec. II A 1, if the global structure of an orbifold contains $n$ fixed points, the compact space exhibits an $S_{n}$ permutation symmetry, which indicates that geometrically all singularities are equivalent. From the perspective of the gauge quantum numbers, fourdimensional effective fields $\Phi_{\ell}$ located at the singularities do not display any difference as long as the gauge embeddings $V_{g}$ associated with the singularities are equal (see Eq. (7) and final remarks in Sec. II B). Under these conditions, the four-dimensional twisted fields build up nontrivial $S_{n}$ representations.

In the case of factorizable orbifolds, i.e., when $\mathbb{T}^{6}$ can be decomposed as $\mathbb{T}^{d_{1}} \times \mathbb{T}^{d_{2}} \times \cdots$, each subtorus has at least a Kähler modulus that allows for differences in the effective theory of the fields originated in different tori. Thus, considering a number of singularities in each torus, the full permutation symmetry of the orbifold is the product $S_{n_{1}} \times S_{n_{2}} \times \cdots$, where each factor corresponds to the permutation symmetry among the fixed points localized at each of the various tori.

Invariance under the full permutation group holds only if all WLs have trivial values. When some WLs are nontrivial, (at least some) twisted states with identical gauge quantum numbers located at various fixed points get different gauge properties and some others do not change. Hence, the fourdimensional field theory is not invariant under the full permutation symmetry anymore, but only under (at most) a (permutation) subgroup thereof. Therefore, the permutation symmetry is said to be explicitly broken by nontrivial WLs in heterotic orbifold compactifications. The permutation symmetry is completely broken when all WLs have nontrivial values.

In order to identify the permutation symmetries, it is important to notice which singularities prevail in the global structure of the orbifold. In simple prime $\mathbb{Z}_{N}$ orbifolds, the same singularities appear in all sectors. However, in less trivial orbifolds, different sectors (corresponding to inequivalent space group elements) have in general different singularities. It is the intersection of all sectors what determine the global structure of the orbifold. This means that only the singularities appearing in all sectors must be regarded to determine the permutation symmetries. These fixed points, which include points in invariant subtori (like those of $\mathbb{Z}_{2} \times \mathbb{Z}_{M}$ orbifolds), exhibit equivalent centralizers and thus the associated twisted states are equal.

Both the permutation symmetry and the Abelian spacegroup symmetries build a large set of symmetry generators, usually denoted by $\left(S_{n_{1}} \times S_{n_{2}} \times \cdots\right) \cup\left(\mathbb{Z}_{N_{1}} \times \mathbb{Z}_{N_{2}} \times \cdots\right)$. The multiplicative closure of the elements of this set constitutes the flavor symmetry perceived by the fourdimensional effective fields. In most cases, the product of Abelian discrete symmetries originated from the space group, $\mathbb{Z}_{N_{1}} \times \mathbb{Z}_{N_{2}} \times \cdots$, is a normal subgroup of $\left(S_{n_{1}} \times S_{n_{2}} \times \cdots\right) \cup\left(\mathbb{Z}_{N_{1}} \times \mathbb{Z}_{N_{2}} \times \cdots\right)$, which implies that the flavor group is given by $G_{F}=\left(S_{n_{1}} \times S_{n_{2}} \times \cdots\right) \ltimes$ $\left(\mathbb{Z}_{N_{1}} \times \mathbb{Z}_{N_{2}} \times \cdots\right)$. Only in a few cases, the resulting symmetry requires extra generators, leading to a symmetry that differs from this structure. This is important when nontrivial WLs are considered.

\section{Flavor symmetries in orbifolds with roto-translations}

If the generators of the space group include rototranslations, some sectors may not exhibit fixed points. As a consequence, no massless states can appear in those sectors and, therefore, the sectors can be ignored to determine the flavor symmetries of the massless spectrum.

As an illustration, let us study again our $\mathbb{T}^{2} / \mathbb{Z}_{2} \times \mathbb{Z}_{2}$ example defined by the generators around Eq. (6). In that case, only the sector $g=g_{1} g_{2}$ has two inequivalent fixed points. The sectors $g_{1}$ and $g_{2}$ do not exhibit fixed points and thus cannot support massless states. The global geometric structure of the orbifold is just that of the projective plane with two singularities, allowing, in the absence of WLs, for an $S_{2} \simeq \mathbb{Z}_{2}$ permutation symmetry of the twisted states. In addition, as we discussed in Sec. III A, there is a $\mathbb{Z}_{2} \times \mathbb{Z}_{2}$ symmetry due to the space group selection rule. That is, we observe that the four-dimensional effective theory must be invariant under the set $S_{2} \cup\left(\mathbb{Z}_{2} \times \mathbb{Z}_{2}\right)$ of symmetries. It is possible to verify that the group $\mathbb{Z}_{2} \times \mathbb{Z}_{2}$ remains invariant under $S_{2}$ elements, so it is a normal subgroup, which implies that the multiplicative closure of the set of symmetries is $S_{2} \ltimes\left(\mathbb{Z}_{2} \times \mathbb{Z}_{2}\right) \simeq D_{4}$. Therefore, the corresponding flavor symmetry is $G_{F}=D_{4}$, which coincides with the emerging flavor symmetry when only one dimension is compactified on an $S^{1} / \mathbb{Z}_{2}$ orbifold.

\section{Flavor symmetries of Abelian orbifolds without Wilson lines}

One of the outcomes of our study is a full classification of the flavor symmetries emerging from six-dimensional Abelian orbifold compactifications without WLs. Interestingly, these symmetries do not depend on the specific string theory to be compactified. They correspond to the flavor symmetries perceived by four-dimensional massless closed-string states attached to the orbifold singularities. Thus, without any further elements (such as D-branes, orientifolds and open strings), the flavor symmetries we find are common to all four-dimensional supersymmetric models arising from orbifold compactifications in generic points of their moduli space.

In these orbifolds, all states associated with fixed points of a particular sector have identical gauge quantum numbers and only their localization in different, independent tori, $T^{d_{1}}, T^{d_{2}}, \ldots$, distinguish them. By applying the 
tools explained in the previous section, we determine the flavor symmetries of all 138 admissible Abelian orbifolds.

Our findings are presented in Table II. Following the notation of Ref. [22], we label each Abelian orbifold, presenting its point group symmetry, and, in parentheses, the labels $(i, j)$ of the corresponding $\mathbb{Z}$ and affine classes, as introduced in Sec. II B. These space group labels are presented in the first and third columns. In the second and fourth columns of Table II, we display the corresponding flavor symmetries at massless level.

There are three space groups which do not lead to any flavor symmetries. The reason is that no fixed points and thus no twisted states appear in those orbifolds. Further, there are 71 orbifolds that yield only Abelian symmetries. The origin of this simplicity in those cases is that only one fixed point is common to all sectors and thus only one point appears in the global structure of the orbifold, avoiding permutation symmetries. We also observe that 45 cases

TABLE II. Flavor symmetries of Abelian toroidal heterotic orbifolds with point groups $\mathbb{Z}_{N}$ and $\mathbb{Z}_{N} \times \mathbb{Z}_{M}$. In the first and third columns, we provide the point group as well as the labels $(i, j)$ of the torus lattice and the roto-translational element, respectively, according to the classification of [22]. The second and fourth columns display the corresponding flavor symmetries. The flavor symmetry for the space group $\mathbb{Z}_{2} \times \mathbb{Z}_{4}(1,1)$ reported in [7] differs from ours because they incorrectly divide an extra $\mathbb{Z}_{2}$.

\begin{tabular}{|c|c|c|}
\hline Orbifold & & Flavor symmetry \\
\hline $\mathbb{Z}_{2} \times \mathbb{Z}_{2}$ & $\begin{array}{l}(1,1) \\
(1,2) \\
(1,3) \\
(1,4) \\
(2,1) \\
(2,2) \\
(2,3) \\
(2,4) \\
(2,5) \\
(2,6) \\
(3,1) \\
(3,2) \\
(3,3) \\
(3,4) \\
(4,1) \\
(4,2) \\
(5,1) \\
(5,2) \\
(5,3) \\
(5,4) \\
(5,5) \\
(6,1) \\
(6,2) \\
(6,3) \\
(7,1) \\
(7,2) \\
(8,1)\end{array}$ & $\begin{array}{c}D_{4}^{6} / \mathbb{Z}_{2}^{4} \\
\mathbb{Z}_{2} \times \mathbb{Z}_{2} \\
\left(D_{4} \times D_{4} \times D_{4}\right) / \mathbb{Z}_{2} \\
\cdots \\
D_{4}^{5} / \mathbb{Z}_{2}^{3} \\
\mathbb{Z}_{2} \times \mathbb{Z}_{2} \\
\left(D_{4} \times D_{4} \times D_{4}\right) / \mathbb{Z}_{2}^{2} \\
\mathbb{Z}_{2} \times \mathbb{Z}_{2} \\
\left(D_{4} \times D_{4}\right) / \mathbb{Z}_{2} \\
\cdots \\
\left(D_{4} \times D_{4} \times D_{4} \times D_{4}\right) / \mathbb{Z}_{2}^{2} \\
\mathbb{Z}_{2} \times \mathbb{Z}_{2} \\
\left(D_{4} \times D_{4}\right) / \mathbb{Z}_{2} \\
\cdots \\
\left(D_{4} \times D_{4} \times D_{4} \times D_{4}\right) / \mathbb{Z}_{2}^{2} \\
\mathbb{Z}_{2} \times \mathbb{Z}_{2} \\
\left(D_{4} \times D_{4} \times D_{4} \times D_{4}\right) / \mathbb{Z}_{2}^{2} \\
\mathbb{Z}_{2} \times \mathbb{Z}_{2} \\
\mathbb{Z}_{2} \times \mathbb{Z}_{2} \\
\left(D_{4} \times D_{4}\right) / \mathbb{Z}_{4} \\
\mathbb{Z}_{2} \times \mathbb{Z}_{2} \\
\left(D_{4} \times D_{4} \times D_{4} \times D_{4}\right) / \mathbb{Z}_{2}^{2} \\
\mathbb{Z}_{2} \times \mathbb{Z}_{2} \\
D_{4} \\
\left(D_{4} \times D_{4} \times D_{4}\right) / \mathbb{Z}_{2} \\
\mathbb{Z}_{2} \times \mathbb{Z}_{2} \\
D_{4} \times D_{4}\end{array}$ \\
\hline
\end{tabular}

(Table continued)
TABLE II. (Continued)

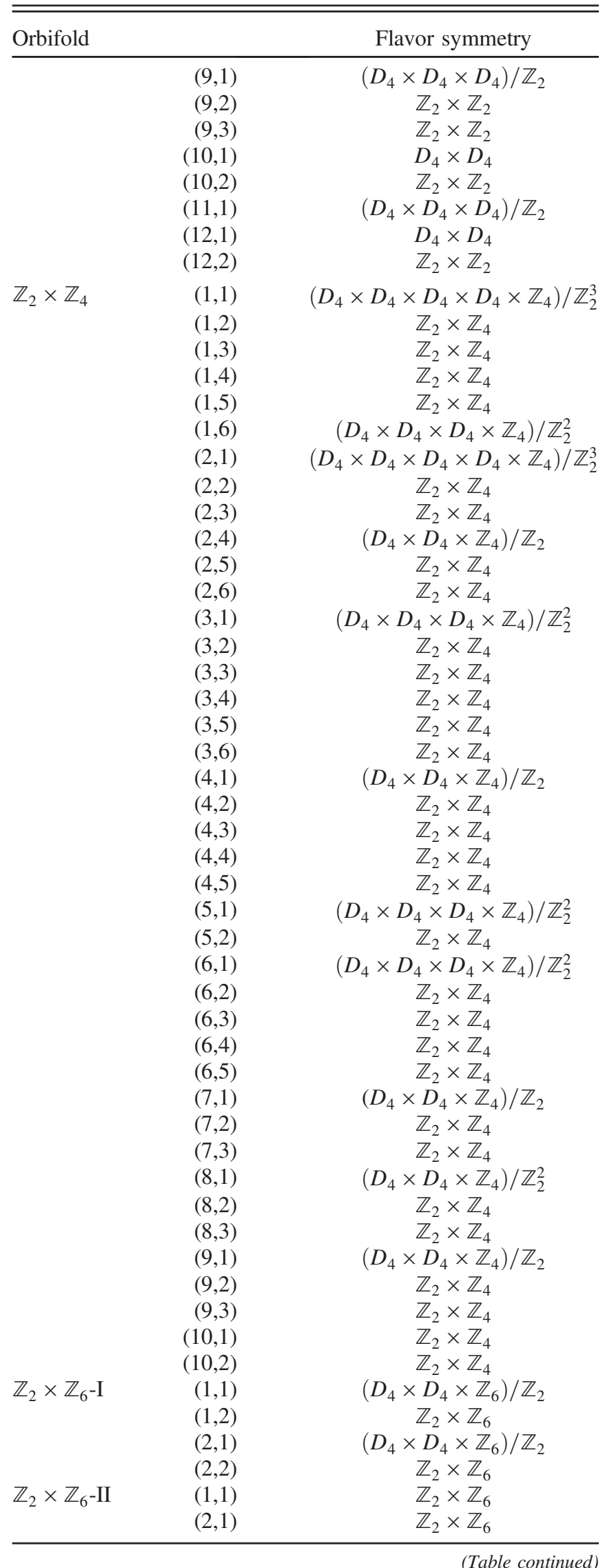


TABLE II. (Continued)

\begin{tabular}{|c|c|c|}
\hline Orbifold & & Flavor symmetry \\
\hline \multirow{17}{*}{$\mathbb{Z}_{3} \times \mathbb{Z}_{3}$} & $(3,1)$ & $\mathbb{Z}_{2} \times \mathbb{Z}_{6}$ \\
\hline & $(4,1)$ & $\mathbb{Z}_{2} \times \mathbb{Z}_{6}$ \\
\hline & $(1,1)$ & $(\Delta(54) \times \Delta(54) \times \Delta(54)) / \mathbb{Z}_{3}$ \\
\hline & $(1,2)$ & $\mathbb{Z}_{3} \times \mathbb{Z}_{3}$ \\
\hline & $(1,3)$ & $\mathbb{Z}_{3} \times \mathbb{Z}_{3}$ \\
\hline & $(1,4)$ & $(\Delta(54) \times \Delta(54)) / \mathbb{Z}_{3}$ \\
\hline & $(2,1)$ & $\Delta(54) \times \Delta(54))$ \\
\hline & $(2,2)$ & $\mathbb{Z}_{3} \times \mathbb{Z}_{3}$ \\
\hline & $(2,3)$ & $\mathbb{Z}_{3} \times \mathbb{Z}_{3}$ \\
\hline & $(2,4)$ & $(\Delta(54) \times \Delta(54)) / \mathbb{Z}_{3}$ \\
\hline & $(3,1)$ & $\Delta(54) \times \Delta(54)$ \\
\hline & $(3,2)$ & $\mathbb{Z}_{3} \times \mathbb{Z}_{3}$ \\
\hline & $(3,3)$ & $(\Delta(54) \times \Delta(54)) / \mathbb{Z}_{3}$ \\
\hline & $(4,1)$ & $\Delta(54) \times \Delta(54)$ \\
\hline & $(4,2)$ & $\mathbb{Z}_{3} \times \mathbb{Z}_{3}$ \\
\hline & $(4,3)$ & $(\Delta(54) \times \Delta(54)) / \mathbb{Z}_{3}$ \\
\hline & $(5,1)$ & $\mathbb{Z}_{3} \times \mathbb{Z}_{3}$ \\
\hline \multirow[t]{4}{*}{$\mathbb{Z}_{3} \times \mathbb{Z}_{6}$} & $(1,1)$ & $\Delta(54) \times \mathbb{Z}_{6}$ \\
\hline & $(1,2)$ & $\mathbb{Z}_{6} \times \mathbb{Z}_{3}$ \\
\hline & $(2,1)$ & $\Delta(54) \times \mathbb{Z}_{6}$ \\
\hline & $(2,2)$ & $\mathbb{Z}_{6} \times \mathbb{Z}_{3}$ \\
\hline \multirow[t]{15}{*}{$\mathbb{Z}_{4} \times \mathbb{Z}_{4}$} & $(1,1)$ & $\left(D_{4} \times D_{4} \times D_{4} \times \mathbb{Z}_{4} \times \mathbb{Z}_{4}\right) / \mathbb{Z}_{2}^{3}$ \\
\hline & $(1,2)$ & $\mathbb{Z}_{4} \times \mathbb{Z}_{4}$ \\
\hline & $(1,3)$ & $\mathbb{Z}_{4} \times \mathbb{Z}_{4}$ \\
\hline & $(1,4)$ & $\mathbb{Z}_{4} \times \mathbb{Z}_{4}$ \\
\hline & $(2,1)$ & $\left(D_{4} \times D_{4} \times \mathbb{Z}_{4}^{2}\right) / \mathbb{Z}_{2}^{2}$ \\
\hline & $(2,2)$ & $\mathbb{Z}_{4} \times \mathbb{Z}_{4}$ \\
\hline & $(2,3)$ & $\mathbb{Z}_{4} \times \mathbb{Z}_{4}$ \\
\hline & $(2,4)$ & $\mathbb{Z}_{4} \times \mathbb{Z}_{4}$ \\
\hline & $(3,1)$ & $\left(D_{4} \times D_{4} \times \mathbb{Z}_{4}^{2}\right) / \mathbb{Z}_{2}^{2}$ \\
\hline & $(3,2)$ & $\mathbb{Z}_{4} \times \mathbb{Z}_{4}$ \\
\hline & $(4,1)$ & $\left(D_{4} \times D_{4} \times \mathbb{Z}_{4}^{2}\right) / \mathbb{Z}_{2}^{2}$ \\
\hline & $(4,2)$ & $\mathbb{Z}_{4} \times \mathbb{Z}_{4}$ \\
\hline & $(4,3)$ & $\mathbb{Z}_{4} \times \mathbb{Z}_{4}$ \\
\hline & $(5,1)$ & $\mathbb{Z}_{4} \times \mathbb{Z}_{4}$ \\
\hline & $(5,2)$ & $\mathbb{Z}_{4} \times \mathbb{Z}_{4}$ \\
\hline $\mathbb{Z}_{6} \times \mathbb{Z}_{6}$ & $(1,1)$ & $\mathbb{Z}_{6} \times \mathbb{Z}_{6}$ \\
\hline $\mathbb{Z}_{3}$ & $(1,1)$ & $(\Delta(54) \times \Delta(54) \times \Delta(54)) / \mathbb{Z}_{3}^{2}$ \\
\hline \multirow[t]{3}{*}{$\mathbb{Z}_{4}$} & $(1,1)$ & $\left(D_{4} \times D_{4} \times D_{4} \times D_{4} \times \mathbb{Z}_{4}\right) / \mathbb{Z}_{2}^{4}$ \\
\hline & $(2,1)$ & $\left(S_{4} \times S_{2} \times S_{2}\right) \ltimes\left(\mathbb{Z}_{4}^{3} \times \mathbb{Z}_{2}^{3}\right)$ \\
\hline & $(3,1)$ & $\left(S_{4} \times S_{4}\right) \ltimes\left(\mathbb{Z}_{4}^{5} \times \mathbb{Z}_{2}^{2}\right)$ \\
\hline \multirow[t]{2}{*}{$\mathbb{Z}_{6}-\mathrm{I}$} & $(1,1)$ & $\Delta(54)$ \\
\hline & $(2,1)$ & $\left(\Delta(54) \times \mathbb{Z}_{6}\right) / \mathbb{Z}_{3}$ \\
\hline \multirow[t]{4}{*}{$\mathbb{Z}_{6}-\mathrm{II}$} & $(1,1)$ & $\Delta(54) \times\left[D_{4} \times D_{4} / \mathbb{Z}_{2}\right]$ \\
\hline & $(2,1)$ & {$\left[\left(\Delta(54) \times \mathbb{Z}_{6}\right) / \mathbb{Z}_{3}\right] \times\left[D_{4}^{2} / \mathbb{Z}_{2}^{2}\right]$} \\
\hline & $(3,1)$ & {$\left[\left(\Delta(54) \times \mathbb{Z}_{6}\right) / \mathbb{Z}_{3}\right] \times\left[D_{4}^{2} / \mathbb{Z}_{2}^{2}\right]$} \\
\hline & $(4,1)$ & {$\left[\left(\Delta(54) \times \mathbb{Z}_{6}\right) / \mathbb{Z}_{3}\right] \times\left[D_{4} / \mathbb{Z}_{2}\right]$} \\
\hline $\mathbb{Z}_{7}$ & $(1,1)$ & $S_{7} \ltimes \mathbb{Z}_{7}^{6}$ \\
\hline \multirow[t]{3}{*}{$\mathbb{Z}_{8}-\mathrm{I}$} & $(1,1)$ & $\left(D_{4} \times D_{4} \times \mathbb{Z}_{8}\right) / \mathbb{Z}_{2}^{2}$ \\
\hline & $(2,1)$ & $\left(D_{4} \times D_{4} \times \mathbb{Z}_{8}\right) / \mathbb{Z}_{2}^{2}$ \\
\hline & $(3,1)$ & $S_{4} \ltimes\left(\mathbb{Z}_{8} \times \mathbb{Z}_{4}^{2} \times \mathbb{Z}_{2}\right)$ \\
\hline
\end{tabular}

(Table continued)
TABLE II. (Continued)

\begin{tabular}{lcc}
\hline \hline Orbifold & & Flavor symmetry \\
\hline $\mathbb{Z}_{8}$-II & $(1,1)$ & $\left(D_{4} \times D_{4} \times D_{4} \times \mathbb{Z}_{8}\right) / \mathbb{Z}_{2}^{3}$ \\
& $(2,1)$ & $\left(D_{4} \times D_{4} \times \mathbb{Z}_{8}\right) / \mathbb{Z}_{2}^{2}$ \\
$\mathbb{Z}_{12}$-I & $(1,1)$ & $\Delta(54)$ \\
& $(2,1)$ & $\left(\Delta(54) \times \mathbb{Z}_{12}\right) / \mathbb{Z}_{3}$ \\
$\mathbb{Z}_{12}$-II & $(1,1)$ & $\left(D_{4} \times D_{4}\right) / \mathbb{Z}_{2}$ \\
\hline \hline
\end{tabular}

include $D_{4}$ factors, whereas 19 space groups lead to $\Delta(54)$ flavor-symmetry factors, three exhibit $S_{4}$ and only one contains $S_{7}$. In some cases the structure of the flavor symmetry follows a factorizable pattern, that is, the resulting flavor symmetry is the direct product of two or more independent non-Abelian symmetries; see e.g., the $(8,1)$ geometry of $\mathbb{Z}_{2} \times \mathbb{Z}_{2}$ orbifolds. However, most of the resulting flavor symmetries are more complicated products and quotients of several permutation and cyclic groups.

As expected from previous studies [3], $D_{4}$ flavor factors appear in $\mathbb{Z}_{2} \times \mathbb{Z}_{M}$ orbifolds whereas $\Delta(54)$ is present in $\mathbb{Z}_{3} \times \mathbb{Z}_{M}$ orbifolds. However, we see that also other symmetries arise in those cases. Thus, only the careful study of the space groups that we carry out here reveals the flavor symmetries of the four-dimensional effective theories arising from orbifold compactifications.

Note that, given a $\mathbb{Z}_{N} \times \mathbb{Z}_{M}$ point group, the largest flavor symmetry arises for $(i, j)=(1,1)$, because the space groups with $i, j>1$ correspond to nonfactorizable six-dimensional tori and/or include roto-translations. Both features reduce the number of fixed points in the orbifold with respect to the $(1,1)$ space group, avoiding large permutation symmetries. Yet there are two exceptional cases: the flavor symmetries of $\mathbb{Z}_{6}-\mathrm{I}$ and $\mathbb{Z}_{12}-\mathrm{I}(1,1)$ orbifolds are smaller than those for $i>1$. This follows from the fact that the point group induces only a $\mathbb{Z}_{3}$ symmetry for the twisted states due to their localization in the $i=1$ case.

One of the conclusions one may draw from these results is that the four-dimensional massless spectrum of supersymmetric heterotic orbifold compactifications can only have one of the flavor symmetries presented here or a subgroup thereof. However, these symmetries may be enhanced by imposing very special conditions on the vacuum, that is, by requiring that the expectation values of moduli satisfy particular relations. For example, if one demanded that all Kähler moduli of the $(1,1)$ case of $\mathbb{Z}_{3} \times \mathbb{Z}_{3}$ have the same value, the $\Delta(54)^{3} / \mathbb{Z}_{3}$ flavor symmetry would be enhanced to the multiplicative closure of $S_{27} \cup \mathbb{Z}_{3}{ }^{5}$.

It has also been shown that the discrete flavor symmetries found here can be enlarged to continuous gauge symmetries at some symmetry-enhanced points of the moduli space [12]. In this work, as already pointed out, we suppose that, if moduli stabilization is possible in these scenarios [51,52], the vacua obtained correspond in general to nonenhanced points in the moduli space. 
TABLE III. Number of $\mathbb{Z}_{N}$ heterotic orbifold models with different geometries yielding the MSSM matter spectrum. In the first column it is shown the orbifold label according to [22]. The maximum number of independent WLs is written in the second column and the number of models found for each number of vanishing WLs is also shown. In the final column we display the total number of MSSM-like models.

\begin{tabular}{|c|c|c|c|c|c|c|c|}
\hline \multirow[b]{3}{*}{ Orbifolc } & & \multirow[b]{3}{*}{ Max \# of independent WLs } & \multicolumn{4}{|c|}{ \# of MSSM-like models with } & \multirow[b]{3}{*}{ Total } \\
\hline & & & 0 & 1 & 2 & 3 & \\
\hline & & & \multicolumn{4}{|c|}{ vanishing WL } & \\
\hline \multirow[t]{2}{*}{$\mathbb{Z}_{4}$} & $(2,1)$ & 3 & 149 & 0 & 0 & 0 & 149 \\
\hline & $(3,1)$ & 2 & 27 & 0 & 0 & & 27 \\
\hline \multirow[t]{2}{*}{$\mathbb{Z}_{6}-\mathrm{I}$} & $(1,1)$ & 1 & 30 & 0 & & & 30 \\
\hline & $(2,1)$ & 1 & 30 & 0 & & & 30 \\
\hline \multirow{4}{*}{$\mathbb{Z}_{6}-\mathrm{II}$} & $(1,1)$ & 3 & 26 & 337 & 0 & 0 & 363 \\
\hline & $(2,1)$ & 3 & 14 & 335 & 0 & 0 & 349 \\
\hline & $(3,1)$ & 3 & 18 & 335 & 0 & 0 & 353 \\
\hline & $(4,1)$ & 2 & 44 & 312 & 0 & & 356 \\
\hline $\mathbb{Z}_{7}$ & $(1,1)$ & 1 & 1 & 0 & & & 1 \\
\hline \multirow[t]{3}{*}{$\mathbb{Z}_{8}-\mathrm{I}$} & $(1,1)$ & 2 & 230 & 38 & 0 & & 268 \\
\hline & $(2,1)$ & 2 & 205 & 41 & 0 & & 246 \\
\hline & $(3,1)$ & 1 & 389 & 0 & & & 389 \\
\hline \multirow[t]{2}{*}{$\mathbb{Z}_{8}-\mathrm{II}$} & $(1,1)$ & 3 & 1,604 & 398 & 21 & 0 & 2,023 \\
\hline & $(2,1)$ & 2 & 274 & 231 & 0 & & 505 \\
\hline \multirow[t]{2}{*}{$\mathbb{Z}_{12}-\mathrm{I}$} & $(1,1)$ & 1 & 556 & 0 & & & 556 \\
\hline & $(2,1)$ & 1 & 555 & 0 & & & 555 \\
\hline $\mathbb{Z}_{12}$-II & $(1,1)$ & 2 & 279 & 84 & 0 & & 363 \\
\hline
\end{tabular}

\section{IV. $\mathbb{Z}_{N} \times \mathbb{Z}_{M}$ HETEROTIC ORBIFOLDS WITH MSSM-LIKE PROPERTIES}

The flavor symmetries classified and showed in Table II correspond to the largest symmetries that the fourdimensional effective field models emerging from orbifold compactifications exhibit. However, in general, they are not the flavor symmetries that models with semirealistic features have because those models include nontrivial WLs, which break the permutation symmetries and thus the flavor symmetries.

To determine the unbroken flavor symmetries that promising models can have, one must know the WLstructure of all promising orbifold compactifications in the different geometries, and, furthermore, the unbroken subgroup of the flavor groups once nontrivial WLs are included in those orbifold geometries.

Clearly, performing a full classification of semirealistic heterotic orbifolds is beyond our capabilities. The first reason is that, given the vastness of the landscape, even the best available algorithms to look for promising models could miss some of them. A second reason is that any such a classification will certainly be very time-consuming. Instead, we use the orbifolder [37] to perform a random search of Abelian orbifold compactifications with properties similar to those of the MSSM. Even in this context, exploring all geometries is very challenging. Thus, since it seems more likely to find appropriate phenomenology with non-Abelian flavor symmetries, we restrict ourselves to a search of phenomenologically promising models, considering only the $64\left(19 \mathbb{Z}_{N}\right.$ and $\left.45 \mathbb{Z}_{N} \times \mathbb{Z}_{M}\right)$ orbifold geometries that allow for non-Abelian flavor symmetries in the absence of WLs (see Table II).

We shall regard here an orbifold compactification as phenomenologically viable if its four-dimensional effective massless spectrum satisfies the following requirements:

(i) the unbroken gauge group is $\mathcal{G}_{\mathrm{SM}} \times \mathcal{G}_{\text {hidden }}=$ $\mathrm{SU}(3)_{c} \times \mathrm{SU}(2)_{L} \times \mathrm{U}(1)_{Y} \times \mathcal{G}_{\text {hidden }}$, where $\mathcal{G}_{\text {hidden }}$ contains additionally (Abelian and non-Abelian) continuous gauge factors, and the $\mathrm{U}(1)_{Y}$ is nonanomalous and compatible with grand unification;

(ii) the effective (twisted and untwisted) states include fields that reproduce the matter spectrum of the MSSM; and

(iii) additional effective states are vector-like with respect to $\mathcal{G}_{\mathrm{SM}}$ and include SM singlets that can play the role of right-handed neutrinos.

With these restrictions, we have performed a broad (although nonexhaustive) search of inequivalent promising models arising from Abelian toroidal orbifold geometries that exhibit non-Abelian flavor symmetries in the absence of WLs. We have studied 19 geometries of $\mathbb{Z}_{N}$ orbifolds and 45 geometries of $\mathbb{Z}_{N} \times \mathbb{Z}_{M}$ orbifolds, including cases with roto-translations. In Tables III and IV, we report the results of our search.

Table III displays the number of $\mathbb{Z}_{N}$ orbifolds models with promising features. There are no models with the 
TABLE IV. Number of $\mathbb{Z}_{N} \times \mathbb{Z}_{M}$ heterotic orbifold models with different geometries yielding the MSSM matter spectrum. The maximum number of independent WLs and the total number of models is also shown. The number of promising models of $\mathbb{Z}_{6} \times \mathbb{Z}_{6}$ orbifolds is presented as a representative of geometries not admitting WLs and yielding four-dimensional field theories endowed only with Abelian flavor symmetries; it is remarkable to find a large number of semirealistic models even without WLs.

\begin{tabular}{|c|c|c|c|c|c|c|c|c|}
\hline \multirow[b]{3}{*}{ Orbifold } & & \multirow[b]{3}{*}{ Max \# of independent WL } & \multicolumn{5}{|c|}{ \# of MSSM-like models with } & \multirow[b]{3}{*}{ Total } \\
\hline & & & 0 & 1 & 2 & 3 & $\geq 4$ & \\
\hline & & & \multicolumn{5}{|c|}{ vanishing WL } & \\
\hline \multirow{10}{*}{$\mathbb{Z}_{2} \times \mathbb{Z}_{2}$} & $(1,1)$ & 6 & 1 & 152 & 52 & 0 & 0 & 205 \\
\hline & $(2,1)$ & 5 & 13 & 342 & 14 & 0 & 0 & 369 \\
\hline & $(3,1)$ & 5 & 4 & 400 & 40 & 0 & 0 & 444 \\
\hline & $(5,1)$ & 4 & 2 & 40 & 0 & 0 & 0 & 42 \\
\hline & $(6,1)$ & 4 & 344 & 57 & 0 & 0 & 0 & 401 \\
\hline & $(7,1)$ & 4 & 21 & 55 & 0 & 0 & 0 & 76 \\
\hline & $(8,1)$ & 4 & 25 & 0 & 0 & 0 & & 25 \\
\hline & $(9,1)$ & 3 & 25 & 2 & 0 & 0 & & 27 \\
\hline & $(10,1)$ & 3 & 19 & 2 & 0 & 0 & & 21 \\
\hline & $(12,1)$ & 2 & 3 & 0 & 0 & & & 3 \\
\hline \multirow[t]{11}{*}{$\mathbb{Z}_{2} \times \mathbb{Z}_{4}$} & $(1,1)$ & 4 & 454 & 8,637 & 1,463 & 26 & 0 & 10,580 \\
\hline & $(1,6)$ & 2 & 65 & 21 & 0 & & & 86 \\
\hline & $(2,1)$ & 4 & 260 & 4,686 & 1,131 & 81 & 0 & 6,158 \\
\hline & $(2,4)$ & 2 & 281 & 47 & 0 & & & 328 \\
\hline & $(3,1)$ & 3 & 13,117 & 3,637 & 103 & 0 & & 16,857 \\
\hline & $(4,1)$ & 3 & 2,911 & 1,575 & 33 & 0 & & 4,519 \\
\hline & $(5,1)$ & 3 & 1,311 & 742 & 63 & 0 & & 2,116 \\
\hline & $(6,1)$ & 3 & 1,814 & 1,374 & 58 & 0 & & 3,246 \\
\hline & $(7,1)$ & 3 & 1,481 & 1,122 & 64 & 0 & & 2,667 \\
\hline & $(8,1)$ & 2 & 839 & 72 & 0 & & & 911 \\
\hline & $(9,1)$ & 2 & 1,620 & 522 & 0 & & & 2,142 \\
\hline \multirow[t]{2}{*}{$\mathbb{Z}_{2} \times \mathbb{Z}_{6}-\mathrm{I}$} & $(1,1)$ & 2 & 467 & 116 & 0 & & & 583 \\
\hline & $(2,1)$ & 2 & 275 & 78 & 0 & & & 353 \\
\hline \multirow[t]{5}{*}{$\mathbb{Z}_{3} \times \mathbb{Z}_{3}$} & $(1,1)$ & 3 & 40 & 987 & 81 & 0 & & 1,108 \\
\hline & $(1,4)$ & 1 & 8 & 0 & & & & 8 \\
\hline & $(2,1)$ & 2 & 1,713 & 239 & 0 & & & 1,952 \\
\hline & $(3,1)$ & 2 & 6 & 0 & 0 & & & 6 \\
\hline & $(4,1)$ & 2 & 105 & 110 & 0 & & & 215 \\
\hline \multirow[t]{2}{*}{$\mathbb{Z}_{3} \times \mathbb{Z}_{6}$} & $(1,1)$ & 1 & 4,469 & 24 & & & & 4,493 \\
\hline & $(2,1)$ & 1 & 495 & 45 & & & & 540 \\
\hline \multirow[t]{4}{*}{$\mathbb{Z}_{4} \times \mathbb{Z}_{4}$} & $(1,1)$ & 3 & 599 & 12,091 & 2,258 & 5 & & 14,953 \\
\hline & $(2,1)$ & 2 & 2,807 & 3,220 & 19 & & & 6,046 \\
\hline & $(3,1)$ & 2 & 2,039 & 875 & 6 & & & 2,920 \\
\hline & $(4,1)$ & 2 & 1,876 & 1,552 & 6 & & & 3,434 \\
\hline $\mathbb{Z}_{6} \times \mathbb{Z}_{6}$ & $(1,1)$ & 0 & 3,412 & & & & & 3,412 \\
\hline
\end{tabular}

required properties of $\mathbb{Z}_{3}$ and $\mathbb{Z}_{4}(1,1)$ orbifolds, reason why these cases are not presented. In the first column, we label each orbifold geometry as in Table II. In the second column, we write the maximal number of admissible WLs. The numbers presented in the third through sixth columns correspond to the number of models with $0,1,2$ and 3 vanishing WLs. For example, we have found 398 semirealistic $\mathbb{Z}_{8}$-II $(1,1)$ orbifold models with one vanishing WL (out of maximally three possible WLs); i.e., there are 398 promising models with two nonzero WLs. The last column provides the total number of models of each geometry. In total, we find 6,563 phenomenologically viable models arising from all $\mathbb{Z}_{N}$ orbifold geometries. We notice that about $52 \%$ of these models arise from the different geometries of $\mathbb{Z}_{8}$ orbifolds.

In Table IV, where the same notation as in Table III is followed, we show our results for the $\mathbb{Z}_{N} \times \mathbb{Z}_{M}$ geometries we selected because they exhibit non-Abelian flavor symmetries in the absence of WLs. Only space groups that yield promising models are presented; this is why only 34 (out of the 45 chosen) geometries are listed. 
Some $\mathbb{Z}_{2} \times \mathbb{Z}_{2}$ orbifold geometries cannot produce MSSM-like models because their structures forbid chiral matter. This was already pointed out in [53,54] as a consequence of the conditions imposed by the space group: in some $\mathbb{Z}_{2} \times \mathbb{Z}_{2}$ orbifolds, all sectors yield independent effective six-dimensional $\mathcal{N}=2$ supersymmetric effective theories, whose combination corresponds to nonchiral field theories from the four-dimensional perspective of the full compactification.

Table IV includes the results for $\mathbb{Z}_{6} \times \mathbb{Z}_{6}$ orbifolds, even though this geometry does not provide non-Abelian flavor symmetries at massless level, solely for the purpose of comparison.

Excluding $\mathbb{Z}_{6} \times \mathbb{Z}_{6}$, we have found $87,834 \mathbb{Z}_{N} \times \mathbb{Z}_{M}$ heterotic orbifold compactifications whose four-dimensional effective theories satisfy our phenomenological constraints. Interestingly, about $56.5 \%$ of these promising models arise from the different geometries of $\mathbb{Z}_{2} \times \mathbb{Z}_{4}$ orbifolds. As expected, most models with the features of the MSSM require nontrivial WLs; however, there are a few $\mathbb{Z}_{3} \times \mathbb{Z}_{6}$ and $\mathbb{Z}_{4} \times \mathbb{Z}_{4}$ examples where the shift vector suffices to render a consistent gauge embedding of the compactification geometry with four-dimensional promising features.

In comparison, we note that $\mathbb{Z}_{6} \times \mathbb{Z}_{6}$ is much more fruitful in this sense. Although $\mathbb{Z}_{6} \times \mathbb{Z}_{6}$ orbifolds do not admit nontrivial WLs, there are thousands of models with MSSM-like properties. This observation may trigger a phenomenological study on heterotic orbifold models with Abelian flavor symmetries.

On the other hand, we find that there are only 422 promising models with roto-translations, which can be identified from Table IV, by inspecting the labels $(i, j)$ : those space groups with $j>1$ include roto-translations. One of the reasons for this behavior is that space groups with roto-translations impose more restrictions on the admissibility of WLs, thus making more difficult the appearance of MSSM-like compactifications.

The list of all $94,397 \mathbb{Z}_{N}$ and $\mathbb{Z}_{N} \times \mathbb{Z}_{M}$ promising orbifold compactifications of the $\mathrm{E}_{8} \times \mathrm{E}_{8}$ heterotic string found in this study is provided in [55], where not only the defining data (as required by the orbifolder) for each of the models is provided, but also their associated flavor symmetries. Although our results are compatible with previous findings $[23,56]$, we find as many as seven times more models than preceding studies. Thus, our results represent the most exhaustive search of semi-realistic string compactifications so far.

\section{FLAVOR SYMMETRIES IN PROMISING STRING COMPACTIFICATIONS}

One purpose of this work is to provide the flavor symmetries that phenomenologically viable Abelian orbifolds admit. Since most of the promising models discussed in the preceding section require nonzero WLs, we investigate now the flavor symmetries that arise when nontrivial
WLs are included in orbifold models with the geometries that led to our promising models.

As stated in Sec. III B, nonzero WLs fully break some of the permutation $S_{n}$ symmetries. If there are independent permutation symmetries, different WLs can break them if they acquire nontrivial values. Consequently, if some WLs have nonvanishing values, the flavor group of the effective model is a (non-Abelian or Abelian) subgroup of the classified flavor symmetries of Table II.

A six-dimensional orbifold compactification can have up to six nontrivial WLs $A_{i}$ of different orders $N_{i}$, but the constraints on the gauge embedding imposed by each space group, discussed in Sec. II B, inhibit nontrivial values for some (and, in some cases, all) of them. For example, in the two-dimensional orbifold introduced in Sec. II A 1, one can verify that the WLs $A_{1}$ and $A_{2}$ associated with the directions $e_{1}$ and $e_{2}$ must be trivial. Thus, if a promising model appeared from such a geometry, its flavor symmetry would then be $D_{4}$. However, many geometries do admit nontrivial WLs. Details of the general properties of the WLs allowed by all Abelian space groups are given in Ref. [22].

We have systematically determined the flavor symmetries that appear once nontrivial WLs are included in the orbifold geometries that allow for non-Abelian flavor symmetries, according to Table II. Our results are presented in Table V for $\mathbb{Z}_{N}$ and Table VI for $\mathbb{Z}_{N} \times \mathbb{Z}_{M}$ orbifold geometries, where only those space groups that allow for at least one WL are shown. In those tables, we label the orbifold geometries according to their space groups, using, as before, the notation of Ref. [22]. After the label, the maximal possible number of inequivalent nontrivial WLs is presented.

In the fourth through seventh columns of Table V, we provide the flavor symmetries that arise in $\mathbb{Z}_{N}$ orbifolds when $\ell=1, \ldots, 4$ nontrivial WLs are allowed. Since some orbifold geometries admit WLs of different orders and/or, even if they have the same order, their action is not symmetric in all compact directions, there may be more than one possible flavor symmetry for the same number of nonvanishing WLs.

For example, consider the space group $\mathbb{Z}_{4}(2,1)$, that admits up to three nontrivial WLs, two of which must have order two and one must be of order four, and yields the flavor group $\left(S_{4} \times S_{2}{ }^{2}\right) \ltimes\left(\mathbb{Z}_{4}{ }^{3} \times \mathbb{Z}_{2}{ }^{3}\right)$. Nontrivial values for an order-2 WL break an $S_{2}$ whereas the order- 4 WL breaks the $S_{4}$ permutation symmetry; that is, if one order-2 WL and one order- 4 WLs are given nontrivial values, the flavor symmetry contains only $S_{2}$ as permutation factor, while if both order-2 WLs acquire nontrivial values, only $S_{4}$ appears. The resulting flavor symmetries in these cases are $S_{2} \ltimes\left(\mathbb{Z}_{4} \times \mathbb{Z}_{2}\right)^{2}$ and $S_{4} \ltimes\left(\mathbb{Z}_{4} \times \mathbb{Z}_{2}\right)^{2}$, respectively. The breakdown of a $\mathbb{Z}_{4} \times$ $\mathbb{Z}_{2}$ factor in the former case is related to the multiplicative closure: it is automatically broken when $S_{4}$ is no longer a symmetry. Both possible flavor symmetries with $\ell=2$ WLs are stacked one over the other in the 
corresponding column of Table V. We repeat this reasoning for all geometries.

Just below each flavor symmetry, we show the number of heterotic orbifold models with phenomenologically appealing properties found in our search with such flavor symmetry (see Sec. IV) and a given number of nontrivial WLs. There are several flavor symmetries with which no promising model can be associated. The final column of the table counts the total of MSSM-like models corresponding to each space group.

Table VI follows a similar notation, but there are some differences. First, in some $\mathbb{Z}_{N} \times \mathbb{Z}_{M}$ orbifold geometries, there are WLs that do not alter the degeneracy of the fixed points even though they do have an impact on the gauge group and other four-dimensional properties of the model. For this reason, we provide in the fourth column the maximal number of WLs that affect the flavor group. Secondly, in some other cases, we find different symmetries for orbifolds with up to $\ell=6$ nontrivial WLs, which are given in the fifth through tenth columns.

As before, we also provide under each flavor symmetry the number of inequivalent promising models found with such symmetries. The total number of phenomenologically viable models is given in the last column. We recall here that, as we already observed in Table IV, there are some MSSM-like models that do not require nontrivial WLs; their flavor symmetries do not appear in Table VI (because they are included in Table II), but they are counted as part of the total number of models.

\section{A. Distribution of flavor symmetries}

Inspecting our results given in Tables V and VI reveals that (excluding the 3,412 models arising from $\mathbb{Z}_{6} \times \mathbb{Z}_{6}$ orbifolds) the 94,397 promising models identified in the previous section have one of three types of flavor symmetries:

(i) Products and quotients of powers of $D_{4}$ with Abelian $\mathbb{Z}_{n}$ factors. We identified as many as 66,742 models with this kind of non-Abelian flavor symmetries, which amount to about $70.5 \%$ of all promising models. The most frequent combination is $D_{4} \times \mathbb{Z}_{4} \times \mathbb{Z}_{2}^{2}$, which arises naturally in $\mathbb{Z}_{2} \times \mathbb{Z}_{4}$ orbifolds.

(ii) Pure Abelian flavor symmetries, including (direct) products of $\mathbb{Z}_{2}, \mathbb{Z}_{3}, \mathbb{Z}_{4}, \mathbb{Z}_{6}, \mathbb{Z}_{7}, \mathbb{Z}_{8}$ and $\mathbb{Z}_{12}$ at different powers. These groups result from the breakdown of all permutation symmetries by the WLs and are thus the symmetries arising from the space-group selection rule. We found 26,189 models of this type, corresponding to about $28 \%$ of the total.

(iii) Products and quotients of powers of $\Delta(54)$ with Abelian $\mathbb{Z}_{n}$ factors. We found only 1,466 models with these flavor symmetries, which represent about $1.5 \%$ of all MSSM-like Abelian heterotic orbifolds we obtained. Models with these flavor symmetries arise only from orbifolds whose space group has a $\mathbb{Z}_{3}$ generator, which are not many.

The defining parameters for our promising models, together with their flavor symmetries, are given in [55].

The fact that $D_{4}$ appears in the majority of our models was expected because we saw already from Table II that most of the space groups yielding non-Abelian flavor symmetries in compactifications without WLs contain $D_{4}$. However, the proportion with respect to models endowed with $\Delta(54)$ is much larger than expected, disfavoring somewhat the latter.

It is known that orbifold compactifications with a $D_{4}$ flavor symmetry and an MSSM-like matter spectrum are such that matter generations split in $\mathbf{2}+\mathbf{1}$ representations of $D_{4}$, where the third generation and its mixings are distinct from the other two, producing some reasonable CKM patterns once the flavor symmetry is broken by VEVs of some SM singlet fields, which turns out to be required by moduli stabilization and decoupling of exotics. Thus, we conclude that most heterotic orbifold compactifications with non-Abelian flavor symmetries follow these patterns, which may deserve further study.

Even though $\Delta(54)$ is not a favored non-Abelian flavor symmetry in our constructions, the number of promising models is still significant and must, therefore, be considered. Phenomenologically, it has been observed that in $\mathbb{Z}_{3} \times \mathbb{Z}_{3}$ heterotic orbifolds furnished with this flavor symmetry, SM generations frequently appear in flavor triplets [8]. This means that these appealing models are endowed with three identical SM generations, justifying the origin of the flavor multiplicity, but, even after spontaneous flavor-symmetry breaking, complicating the explanation of the observed quark and neutrino mixing patterns. It might be interesting to investigate whether and how this situation is improved in some other models with this symmetry.

We finally observe that we find that almost no space group with roto-translations leads to MSSM-like models. Only the orbifold geometries $\mathbb{Z}_{2} \times \mathbb{Z}_{4}(1,6), \mathbb{Z}_{2} \times \mathbb{Z}_{4}(2,4)$ and $\mathbb{Z}_{3} \times \mathbb{Z}_{3}(1,4)$ include roto-translations and yield promising models, although the models of $\mathbb{Z}_{3} \times \mathbb{Z}_{3}(1,4)$ admit only Abelian flavor symmetries. One could therefore argue that roto-translations impose generally too tight constraints to arrive at promising Abelian orbifolds. It would be nevertheless interesting to know what kind of phenomenology is produced by these models.

\section{FINAL REMARKS}

With the goal in mind of finding in string theory some guidance principle that singles out the discrete symmetry that might govern the mixing patterns of fermions in the SM, we have investigated the flavor symmetries that arise from compactifying symmetrically the heterotic string on Abelian toroidal orbifolds.

First, we have classified the flavor symmetries associated with the geometry of all admissible Abelian toroidal 
orbifolds. This classification, presented in Table II, is valid for the massless closed-string sector of all string theories compactified on orbifolds for arbitrary values of their moduli. We find that 64 out of 138 admissible space groups yield non-Abelian flavor symmetries, where products of $D_{4}, \Delta(54), S_{4}, S_{7}$ and cyclic groups appear. 71 space groups lead to purely Abelian flavor symmetries and we find no flavor in three cases.

In most cases, arriving at four-dimensional models that reproduce properties of particle physics requires additional elements (such as D-branes, orientifols, Wilson lines, etc.) that break the flavor symmetries we have classified to their subgroups. In the heterotic strings compactified on Abelian toroidal orbifolds, their gauge embeddings admit the inclusion of Wilson lines, whose different values, restricted by modular invariance, lead to a variety of effective field theories.

We have performed the widest known search of MSSMlike Abelian toroidal orbifolds of the $\mathrm{E}_{8} \times \mathrm{E}_{8}$ heterotic string and found more than 94,000 promising models with different properties arising from orbifolds defined by the 64 space groups that yield non-Abelian flavor symmetries in our classification. Almost 88,000 models arise from different geometries of $\mathbb{Z}_{N} \times \mathbb{Z}_{M}$ orbifolds, but only 422 arise from orbifolds with roto-translations, disfavoring this class of models for phenomenology. We show a summary of these results in Tables III and IV. These models represent as many as seven times more models than those found in the literature.

Assuming that these models can describe some generic properties of the region of the string landscape where the stringy ultraviolet completion of the SM resides, we have then studied the flavor properties of these models. We have found that about $70.5 \%$ of them exhibit flavor symmetries that are products of powers of $D_{4}$ and cyclic groups, whereas only about $1.5 \%$ contain $\Delta(54)$. The remaining models are furnished with purely Abelian flavor symmetries, whose origin are the rules that dictate how string states couple after compactification [see Eqs. (14) and (15)]. These results are summarized in the Appendix, and all model definitions are provided in our website [55], where our promising models are classified according to their space group, number of Wilson lines and flavor symmetry.

Two observations of these results are in order. Since models with $D_{4}$ flavor distinguish the third generation from the other two while models with $\Delta(54)$ frequently assign equal properties to all three generations, one can argue that our findings disfavor statistically the second scenario. Nonetheless, one may also be interested in studying the properties of the almost 1,500 models with $\Delta(54)$ as flavor structure.
Our second observation concerns the models with purely Abelian symmetries. It is somewhat surprising that almost one third of our promising models have such flavor symmetries. Further, we must point out that we have not performed a search of MSSM-like constructions for the 71 orbifold space groups that led to Abelian symmetries in the absence of WLs, according to Table II. We have only explored $\mathbb{Z}_{6} \times \mathbb{Z}_{6}$ and found more than 3,000 models endowed with a $\mathbb{Z}_{6} \times \mathbb{Z}_{6}$ flavor symmetry. Extrapolating this number of models, it is conceivable that most promising models arising from orbifold compactifications exhibit Abelian flavor symmetries. Therefore, we consider necessary to further investigate the phenomenological implications of models whose flavor structure coincide with the cyclic symmetry groups we find. We shall pursue this goal elsewhere.

On the other hand, although restricting ourselves to the massless sector of our models is reasonable, the massive sectors of the compactification can also influence physics at low energies. Thus, beside further research on the phenomenology of the models we have found and possible extensions to string compactifications without supersymmetry, one should study how our findings are altered when massive strings or, in other words, all space group elements are considered. This is matter of ongoing research [50].

Additionally, it is well-known that target-space modular symmetries act nontrivially on quarks and leptons arising from orbifold compactifications [57-60]. It has been recently emphasized the key role that these symmetries may play in flavor phenomenology $[11,61-64]$. We find interesting to study systematically these symmetries in heterotic orbifolds, as shall be done elsewhere.

Finally, one can extend the study of the stringy landscape of flavor physics by exploring non-Abelian orbifolds. Since the space group selection rule would most likely lead directly to non-Abelian symmetries, instead of only products of cyclic groups, one could expect a richer flavor structure. Following recent progress on the understanding of these constructions [54], we can pursue this enterprise now.

\section{ACKNOWLEDGMENTS}

It is a pleasure to thank Patrick K. S. Vaudrevange and Hans-Peter Nilles for useful discussions. S. R.-S is grateful to the Bethe Center for Theoretical Physics and the Mainz Institute for Theoretical Physics for the hospitality during the final stage of this work. This work was partly supported by DGAPA-PAPIIT Grant No. IN100217 and CONACyT Grants No. F-252167 and No. 278017. 


\section{APPENDIX: FLAVOR SYMMETRIES IN MODELS WITH WILSON LINES}

TABLE V. Flavor symmetries in $\mathbb{Z}_{N} 6 \mathrm{D}$ orbifolds with WLs. The orbifold labels correspond to the labels of the associated space group, according to Ref. [22]. For each space group yielding non-Abelian flavor symmetries in the absence of WLs (see Table II), we show all possible breakings for non-vanishing WLs. There are cases where the same space group geometry and number of WLs lead to different flavor symmetries; these symmetries are stacked in different rows. Under each flavor symmetry, we show the number of phenomenologically viable heterotic models obtained from our fairly exhaustive search of models (see Sec. IV).

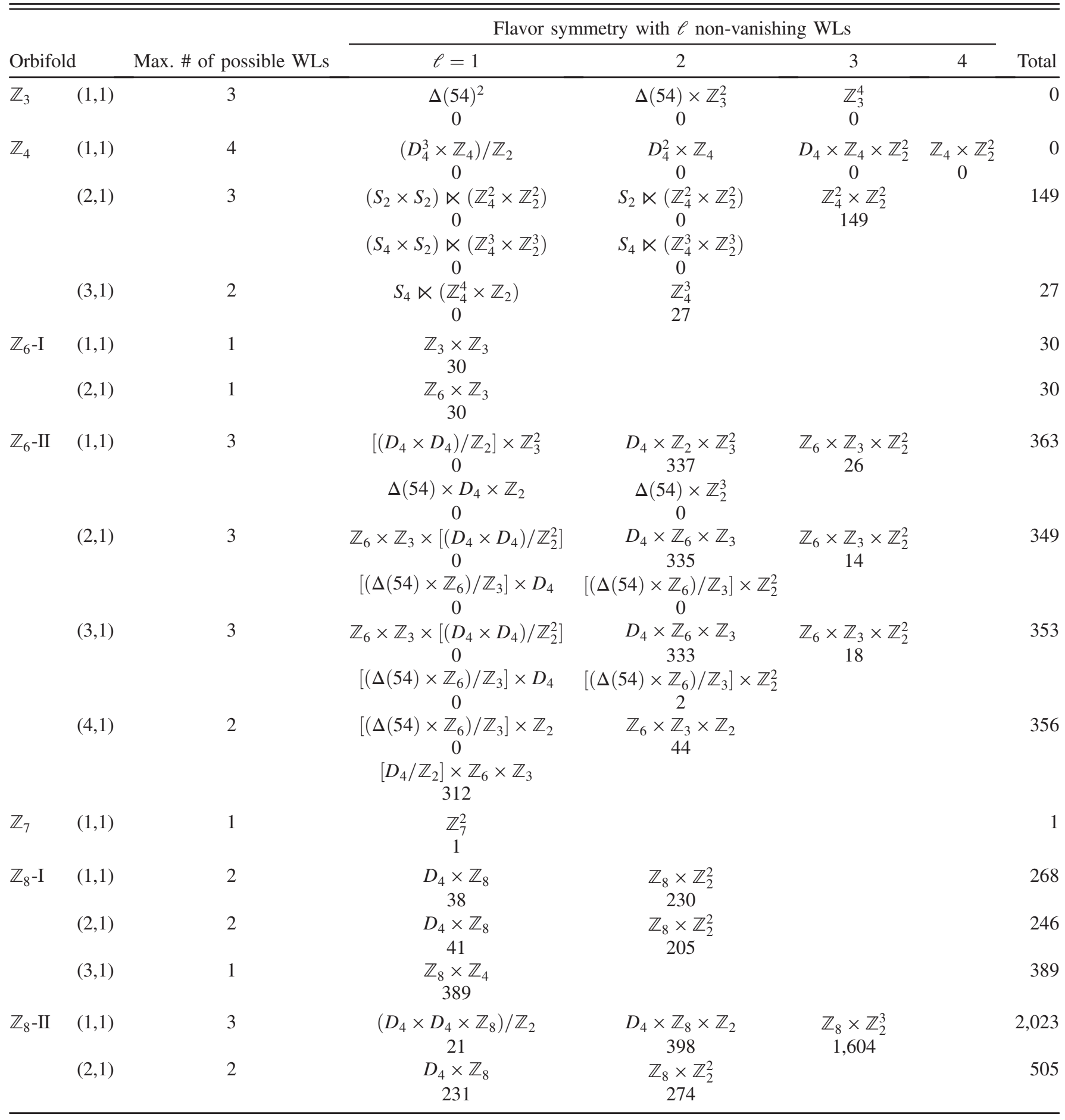


TABLE V. (Continued)

\begin{tabular}{|c|c|c|c|c|c|c|c|}
\hline \multirow{2}{*}{\multicolumn{2}{|c|}{ Orbifold }} & \multirow{3}{*}{$\frac{\text { Max. \# of possible WLs }}{1}$} & \multicolumn{4}{|c|}{ Flavor symmetry with $\ell$ non-vanishing WLs } & \multirow{3}{*}{$\frac{\text { Total }}{556}$} \\
\hline & & & $\ell=1$ & 2 & 3 & 4 & \\
\hline \multirow[t]{2}{*}{$\mathbb{Z}_{12}-\mathrm{I}$} & $(1,1)$ & & $\begin{array}{c}\mathbb{Z}_{3} \times \mathbb{Z}_{3} \\
556\end{array}$ & & & & \\
\hline & $(2,1)$ & 1 & $\begin{array}{c}\mathbb{Z}_{12} \times \mathbb{Z}_{3} \\
555\end{array}$ & & & & 555 \\
\hline $\mathbb{Z}_{12}$-II & $(1,1)$ & 2 & $\begin{array}{c}D_{4} \times \mathbb{Z}_{2} \\
84\end{array}$ & $\begin{array}{c}\mathbb{Z}_{2}^{3} \\
279\end{array}$ & & & 363 \\
\hline
\end{tabular}

TABLE VI. Flavor symmetries in $\mathbb{Z}_{N} \times \mathbb{Z}_{M}$ orbifolds with WLs. The orbifold labels correspond to the labels of the associated space group, according to Ref. [22]. For each space group yielding non-Abelian flavor symmetries in the absence of WLs (see Table II), we show all possible breakings for non-vanishing WLs. There are cases where the same space group geometry and number of WLs lead to different flavor symmetries; these symmetries are stacked in different rows. Under each flavor symmetry, we show the number of phenomenologically viable models obtained from our fairly exhaustive search of models (see Sec. IV). Since some WLs do not break the flavor group, we give in the fourth column the maximal number of WLs that affect the flavor symmetries. For $\mathbb{Z}_{3} \times \mathbb{Z}_{6}$ and $\mathbb{Z}_{4} \times \mathbb{Z}_{4}$ we also count in the total the promising models arising without WLs.

\begin{tabular}{|c|c|c|c|c|c|c|c|c|c|c|}
\hline \multirow[b]{2}{*}{ Orbifold } & & \multirow{2}{*}{$\begin{array}{l}\text { Max. \# } \\
\text { of possible } \\
\text { WLs }\end{array}$} & \multirow{2}{*}{$\begin{array}{l}\text { Max. \# of WLs } \\
\text { affecting the } \\
\text { flavor symmetry }\end{array}$} & \multicolumn{6}{|c|}{ Flavor symmetry with $\ell$ non-vanishing WLs } & \multirow[b]{2}{*}{ Total } \\
\hline & & & & $\ell=1$ & 2 & 3 & 4 & 5 & 6 & \\
\hline \multirow[t]{8}{*}{$\mathbb{Z}_{2} \times \mathbb{Z}_{2}$} & $(1,1)$ & 6 & 6 & $\begin{array}{c}D_{4}^{5} / \mathbb{Z}_{2}^{2} \\
0\end{array}$ & $\begin{array}{c}D_{4}^{4} \\
0\end{array}$ & $\begin{array}{c}D_{4}^{3} \times \mathbb{Z}_{2}^{2} \\
0\end{array}$ & $\begin{array}{c}D_{4}^{2} \times \mathbb{Z}_{2}^{4} \\
52\end{array}$ & $\begin{array}{c}D_{4} \times \mathbb{Z}_{2}^{6} \\
152\end{array}$ & $\begin{array}{c}\mathbb{Z}_{2}^{8} \\
1\end{array}$ & 205 \\
\hline & $(1,3)$ & 4 & 2 & $\begin{array}{c}D_{4}^{2} \\
0 \\
D_{4}^{3} / \mathbb{Z}_{2}^{2} \\
0\end{array}$ & $\begin{array}{c}D_{4} \times \mathbb{Z}_{2}^{2} \\
0 \\
D_{4}^{2} \\
0 \\
D_{4}^{3} / \mathbb{Z}_{2}^{2} \\
0\end{array}$ & $\begin{array}{c}D_{4} \times \mathbb{Z}_{2}^{2} \\
0 \\
D_{4}^{2} \\
0\end{array}$ & $\begin{array}{c}D_{4} \times \mathbb{Z}_{2}^{2} \\
0\end{array}$ & & & 0 \\
\hline & $(2,1)$ & 5 & 5 & $\begin{array}{c}D_{4}^{4} / \mathbb{Z}_{2} \\
0\end{array}$ & $\begin{array}{c}D_{4}^{3} \times \mathbb{Z}_{2} \\
0\end{array}$ & $\begin{array}{c}D_{4}^{2} \times \mathbb{Z}_{2}^{3} \\
14\end{array}$ & $\begin{array}{c}D_{4} \times \mathbb{Z}_{2}^{5} \\
342\end{array}$ & $\begin{array}{l}\mathbb{Z}_{2}^{7} \\
13\end{array}$ & & 369 \\
\hline & $(2,3)$ & 3 & 2 & $\begin{array}{c}D_{4} \times \mathbb{Z}_{2}^{2} \\
0 \\
D_{4}^{2} \\
0 \\
D_{4}^{3} / \mathbb{Z}_{2}^{2} \\
0\end{array}$ & $\begin{array}{c}\mathbb{Z}_{2}^{4} \\
0 \\
D_{4} \times \mathbb{Z}_{2}^{2} \\
0 \\
D_{4}^{2} \\
0\end{array}$ & $\begin{array}{c}\mathbb{Z}_{2}^{4} \\
0\end{array}$ & & & & 0 \\
\hline & $(2,5)$ & 3 & 1 & $\begin{array}{c}D_{4} \times \mathbb{Z}_{2} \\
0 \\
D_{4}^{2} / \mathbb{Z}_{2} \\
0\end{array}$ & $\begin{array}{c}D_{4} \times \mathbb{Z}_{2} \\
0 \\
D_{4}^{2} / \mathbb{Z}_{2} \\
0\end{array}$ & $\begin{array}{c}D_{4} \times \mathbb{Z}_{2} \\
0\end{array}$ & & & & 0 \\
\hline & $(3,1)$ & 5 & 5 & $\begin{array}{c}D_{4}^{3} \\
0\end{array}$ & $\begin{array}{c}D_{4}^{2} \times \mathbb{Z}_{2}^{2} \\
0 \\
D_{4}^{3} \\
0\end{array}$ & $\begin{array}{c}D_{4} \times \mathbb{Z}_{2}^{4} \\
40 \\
D_{4}^{2} \times \mathbb{Z}_{2}^{2} \\
0\end{array}$ & $\begin{array}{c}\mathbb{Z}_{2}^{6} \\
8 \\
D_{4} \times \mathbb{Z}_{2}^{4} \\
392\end{array}$ & $\begin{array}{c}\mathbb{Z}_{2}^{6} \\
4\end{array}$ & & 444 \\
\hline & $(3,3)$ & 3 & 1 & $\begin{array}{c}D_{4} \times \mathbb{Z}_{2} \\
0 \\
D_{4}^{2} / \mathbb{Z}_{2} \\
0\end{array}$ & $\begin{array}{c}D_{4} \times \mathbb{Z}_{2} \\
0 \\
D_{4}^{2} / \mathbb{Z}_{2} \\
0\end{array}$ & $\begin{array}{c}D_{4} \times \mathbb{Z}_{2} \\
0\end{array}$ & & & & 0 \\
\hline & $(4,1)$ & 4 & 2 & $\begin{array}{c}D_{4}^{3} \\
0 \\
D_{4} / \mathbb{Z}_{2}^{2} \\
0\end{array}$ & $\begin{array}{c}D_{4}^{2} \times \mathbb{Z}_{2}^{2} \\
0 \\
D_{4}^{3} \\
0 \\
D_{4} / \mathbb{Z}_{2}^{2} \\
0\end{array}$ & $\begin{array}{c}D_{4}^{2} \times \mathbb{Z}_{2}^{2} \\
0 \\
D_{4}^{3} \\
0\end{array}$ & $\begin{array}{c}D_{4}^{2} \times \mathbb{Z}_{2}^{2} \\
0\end{array}$ & & & 0 \\
\hline
\end{tabular}


TABLE VI. (Continued)

\begin{tabular}{|c|c|c|c|c|c|c|c|c|c|c|}
\hline \multirow[b]{2}{*}{ Orbifold } & & \multirow{2}{*}{$\begin{array}{l}\text { Max. \# } \\
\text { of possible } \\
\text { WLs }\end{array}$} & \multirow{2}{*}{$\begin{array}{l}\text { Max. \# of WLs } \\
\text { affecting the } \\
\text { flavor symmetry }\end{array}$} & \multicolumn{6}{|c|}{ Flavor symmetry with $\ell$ non-vanishing WLs } & \multirow[b]{2}{*}{ Total } \\
\hline & & & & $\ell=1$ & 2 & 3 & 4 & 5 & 6 & \\
\hline & $(5,1)$ & 4 & 4 & $\begin{array}{c}D_{4}^{3} \\
0\end{array}$ & $\begin{array}{c}D_{4}^{2} \times \mathbb{Z}_{2}^{2} \\
0\end{array}$ & $\begin{array}{c}D_{4} \times \mathbb{Z}_{2}^{4} \\
40\end{array}$ & $\begin{array}{c}\mathbb{Z}_{2}^{6} \\
2\end{array}$ & & & 42 \\
\hline & $(5,4)$ & 2 & 1 & $\begin{array}{c}D_{4} \times \mathbb{Z}_{2} \\
0 \\
D_{4}^{2} / \mathbb{Z}_{2} \\
0\end{array}$ & $\begin{array}{c}D_{4} \times \mathbb{Z}_{2} \\
0\end{array}$ & & & & & 0 \\
\hline & $(6,1)$ & 4 & 2 & $\begin{array}{c}D_{4}^{3} \\
0 \\
D_{4}^{2} / \mathbb{Z}_{2}^{2} \\
0\end{array}$ & $\begin{array}{c}D_{4}^{2} \times \mathbb{Z}_{2}^{2} \\
0 \\
D_{4}^{3} \\
0 \\
D_{4}^{4} / \mathbb{Z}_{2}^{2} \\
0\end{array}$ & $\begin{array}{c}D_{4}^{2} \times \mathbb{Z}_{2}^{2} \\
57 \\
D_{4}^{3} \\
0\end{array}$ & $\begin{array}{c}D_{4}^{2} \times \mathbb{Z}_{2}^{2} \\
344\end{array}$ & & & 401 \\
\hline & $(6,3)$ & 2 & 0 & $\begin{array}{c}D_{4} \\
0\end{array}$ & $\begin{array}{c}D_{4} \\
0\end{array}$ & & & & & 0 \\
\hline & $(7,1)$ & 4 & 3 & $\begin{array}{c}D_{4}^{2} \times \mathbb{Z}_{2} \\
0 \\
D_{4}^{3} / \mathbb{Z}_{2} \\
0\end{array}$ & $\begin{array}{c}D_{4} \times \mathbb{Z}_{2}^{3} \\
0 \\
D_{4}^{2} \times \mathbb{Z}_{2} \\
0\end{array}$ & $\begin{array}{c}D_{4} \times \mathbb{Z}_{2}^{3} \\
55 \\
D_{4}^{2} \times \mathbb{Z}_{2} \\
0\end{array}$ & $\begin{array}{c}D_{4} \times \mathbb{Z}_{2}^{3} \\
21\end{array}$ & & & 76 \\
\hline & $(8,1)$ & 4 & 4 & $\begin{array}{c}D_{4} \times \mathbb{Z}_{2}^{2} \\
0\end{array}$ & $\begin{array}{c}\mathbb{Z}_{2}^{4} \\
0 \\
D_{4} \times \mathbb{Z}_{2}^{2} \\
0\end{array}$ & $\begin{array}{c}\mathbb{Z}_{2}^{4} \\
0\end{array}$ & $\begin{array}{l}\mathbb{Z}_{2}^{4} \\
25\end{array}$ & & & 25 \\
\hline & $(9,1)$ & 3 & 2 & $\begin{array}{c}D_{4}^{2} \times \mathbb{Z}_{2} \\
0 \\
D_{4}^{3} / \mathbb{Z}_{2} \\
0\end{array}$ & $\begin{array}{c}D_{4} \times \mathbb{Z}_{2}^{3} \\
2 \\
D_{4}^{2} \times \mathbb{Z}_{2} \\
0\end{array}$ & $\begin{array}{c}D_{4} \times \mathbb{Z}_{2}^{3} \\
25\end{array}$ & & & & 27 \\
\hline & $(10,1)$ & 3 & 3 & $\begin{array}{c}D_{4} \times \mathbb{Z}_{2}^{2} \\
0\end{array}$ & $\begin{array}{c}\mathbb{Z}_{2}^{4} \\
2 \\
D_{4} \times \mathbb{Z}_{2}^{2} \\
0\end{array}$ & $\begin{array}{l}\mathbb{Z}_{2}^{4} \\
19\end{array}$ & & & & 21 \\
\hline & $(11,1)$ & 3 & 0 & $\begin{array}{c}D_{4}^{3} / \mathbb{Z}_{2} \\
0\end{array}$ & $\begin{array}{c}D_{4}^{3} / \mathbb{Z}_{2} \\
0\end{array}$ & $\begin{array}{c}D_{4}^{3} / \mathbb{Z}_{2} \\
0\end{array}$ & & & & 0 \\
\hline & $(12,1)$ & 2 & 2 & $\begin{array}{c}D_{4} \times \mathbb{Z}_{2}^{2} \\
0\end{array}$ & $\begin{array}{c}\mathbb{Z}_{2}^{4} \\
3\end{array}$ & & & & & 3 \\
\hline \multirow[t]{6}{*}{$\mathbb{Z}_{2} \times \mathbb{Z}_{4}$} & $(1,1)$ & 4 & 4 & $\begin{array}{c}\left(D_{4}^{3} \times \mathbb{Z}_{4}\right) / \mathbb{Z}_{2} \\
26\end{array}$ & $\begin{array}{c}D_{4}^{2} \times \mathbb{Z}_{4} \times \mathbb{Z}_{2} \\
1,463\end{array}$ & $\begin{array}{c}D_{4} \times \mathbb{Z}_{4} \times \mathbb{Z}_{2}^{3} \\
8,637\end{array}$ & $\begin{array}{c}\mathbb{Z}_{4} \times \mathbb{Z}_{2}^{5} \\
454\end{array}$ & & & 10,580 \\
\hline & $(1,6)$ & 2 & 2 & $\begin{array}{c}D_{4}^{2} \times \mathbb{Z}_{4} \\
21\end{array}$ & 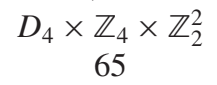 & & & & & 86 \\
\hline & $(2,1)$ & 4 & 4 & $\begin{array}{c}\left(D_{4}^{3} \times \mathbb{Z}_{4}\right) / \mathbb{Z}_{2} \\
81\end{array}$ & $\begin{array}{c}D_{4}^{2} \times \mathbb{Z}_{4} \times \mathbb{Z}_{2} \\
1,131\end{array}$ & $\begin{array}{c}D_{4} \times \mathbb{Z}_{4} \times \mathbb{Z}_{2}^{3} \\
4,686\end{array}$ & $\begin{array}{c}\mathbb{Z}_{4} \times \mathbb{Z}_{2}^{5} \\
260\end{array}$ & & & 6,158 \\
\hline & $(2,4)$ & 2 & 2 & $\begin{array}{c}D_{4} \times \mathbb{Z}_{4} \times \mathbb{Z}_{2} \\
47\end{array}$ & $\begin{array}{c}\mathbb{Z}_{4} \times \mathbb{Z}_{2}^{3} \\
281\end{array}$ & & & & & 328 \\
\hline & $(3,1)$ & 3 & 2 & $\begin{array}{c}D_{4}^{2} \times \mathbb{Z}_{4} \\
27 \\
\left(D_{4}^{3} \times \mathbb{Z}_{4}\right) / \mathbb{Z}_{2}^{2} \\
76\end{array}$ & $\begin{array}{c}D_{4} \times \mathbb{Z}_{4} \times \mathbb{Z}_{2}^{2} \\
1,012 \\
D_{4}^{2} \times \mathbb{Z}_{4} \\
2,625\end{array}$ & $\begin{array}{c}D_{4} \times \mathbb{Z}_{4} \times \mathbb{Z}_{2}^{2} \\
13,117\end{array}$ & & & & 16,857 \\
\hline & $(4,1)$ & 3 & 3 & $\begin{array}{c}D_{4} \times \mathbb{Z}_{4} \times \mathbb{Z}_{2} \\
33\end{array}$ & $\begin{array}{c}\mathbb{Z}_{4} \times \mathbb{Z}_{2}^{3} \\
1,133 \\
D_{4} \times \mathbb{Z}_{4} \times \mathbb{Z}_{2} \\
442\end{array}$ & $\begin{array}{c}\mathbb{Z}_{4} \times \mathbb{Z}_{2}^{3} \\
2,911\end{array}$ & & & & 4,519 \\
\hline
\end{tabular}


TABLE VI. (Continued)

\begin{tabular}{|c|c|c|c|c|c|c|c|c|c|c|}
\hline \multirow[b]{2}{*}{ Orbifold } & & \multirow{2}{*}{$\begin{array}{l}\text { Max. \# } \\
\text { of possible } \\
\text { WLs }\end{array}$} & \multirow{2}{*}{$\begin{array}{l}\text { Max. \# of WLs } \\
\text { affecting the } \\
\text { flavor symmetry }\end{array}$} & \multicolumn{6}{|c|}{ Flavor symmetry with $\ell$ non-vanishing WLs } & \multirow[b]{2}{*}{ Total } \\
\hline & & & & $\ell=1$ & 2 & 3 & 4 & 5 & 6 & \\
\hline & $(5,1)$ & 3 & 2 & $\begin{array}{c}D_{4}^{2} \times \mathbb{Z}_{4} \\
18 \\
\left(D_{4}^{3} \times \mathbb{Z}_{4}\right) / \mathbb{Z}_{2}^{2} \\
45\end{array}$ & $\begin{array}{c}D_{4} \times \mathbb{Z}_{4} \times \mathbb{Z}_{2}^{2} \\
45 \\
D_{4}^{2} \times \mathbb{Z}_{4} \\
697\end{array}$ & $\begin{array}{c}D_{4} \times \mathbb{Z}_{4} \times \mathbb{Z}_{2}^{2} \\
1,311\end{array}$ & & & & 2,116 \\
\hline & $(6,1)$ & 3 & 2 & $\begin{array}{c}D_{4} \times \mathbb{Z}_{4} \times \mathbb{Z}_{2}^{2} \\
18 \\
D_{4}^{2} \times \mathbb{Z}_{4} \\
3 \\
\left(D_{4}^{3} \times \mathbb{Z}_{4}\right) / \mathbb{Z}_{2} \\
37\end{array}$ & $\begin{array}{c}\mathbb{Z}_{4} \times \mathbb{Z}_{2}^{4} \\
511 \\
D_{4} \times \mathbb{Z}_{4} \times \mathbb{Z}_{2}^{2} \\
295 \\
D_{4}^{2} \times \mathbb{Z}_{4} \\
568\end{array}$ & $\begin{array}{c}\mathbb{Z}_{4} \times \mathbb{Z}_{2}^{4} \\
1,814\end{array}$ & & & & 3,246 \\
\hline & $(7,1)$ & 3 & 3 & $\begin{array}{c}D_{4} \times \mathbb{Z}_{4} \times \mathbb{Z}_{2} \\
64\end{array}$ & $\begin{array}{c}\mathbb{Z}_{4} \times \mathbb{Z}_{2}^{3} \\
729 \\
D_{4} \times \mathbb{Z}_{4} \times \mathbb{Z}_{2} \\
393\end{array}$ & $\begin{array}{c}\mathbb{Z}_{4} \times \mathbb{Z}_{2}^{3} \\
1,481\end{array}$ & & & & 2,667 \\
\hline & $(8,1)$ & 2 & 0 & $\begin{array}{c}\left(D_{4}^{2} \times \mathbb{Z}_{4}\right) / \mathbb{Z}_{2} \\
72\end{array}$ & $\begin{array}{c}\left(D_{4}^{2} \times \mathbb{Z}_{4}\right) / \mathbb{Z}_{2} \\
839\end{array}$ & & & & & 911 \\
\hline & $(9,1)$ & 2 & 2 & $\begin{array}{c}D_{4} \times \mathbb{Z}_{4} \times \mathbb{Z}_{2} \\
522\end{array}$ & $\begin{array}{c}\mathbb{Z}_{4} \times \mathbb{Z}_{2}^{3} \\
1,620\end{array}$ & & & & & 2,142 \\
\hline \multirow[t]{2}{*}{$\mathbb{Z}_{2} \times \mathbb{Z}_{6}-\mathrm{I}$} & $(1,1)$ & 2 & 2 & $\begin{array}{c}D_{4} \times \mathbb{Z}_{2} \times \mathbb{Z}_{6} \\
116\end{array}$ & $\begin{array}{c}\mathbb{Z}_{2}^{3} \times \mathbb{Z}_{6} \\
467\end{array}$ & & & & & 583 \\
\hline & $(2,1)$ & 2 & 2 & $\begin{array}{c}D_{4} \times \mathbb{Z}_{2} \times \mathbb{Z}_{6} \\
78\end{array}$ & $\begin{array}{c}\mathbb{Z}_{2}^{3} \times \mathbb{Z}_{6} \\
275\end{array}$ & & & & & 353 \\
\hline \multirow[t]{5}{*}{$\mathbb{Z}_{3} \times \mathbb{Z}_{3}$} & $(1,1)$ & 3 & 3 & $\begin{array}{c}\Delta(54)^{2} \times \mathbb{Z}_{3} \\
81\end{array}$ & $\begin{array}{c}\Delta(54) \times \mathbb{Z}_{3}^{3} \\
987\end{array}$ & $\begin{array}{l}\mathbb{Z}_{3}^{5} \\
40\end{array}$ & & & & 1,108 \\
\hline & $(1,4)$ & 1 & 1 & $\begin{array}{c}\mathbb{Z}_{3}^{3} \\
8\end{array}$ & & & & & & 8 \\
\hline & $(2,1)$ & 2 & 2 & $\begin{array}{c}\Delta(54) \times \mathbb{Z}_{3}^{2} \\
239\end{array}$ & $\begin{array}{c}\mathbb{Z}_{3}^{4} \\
1,713\end{array}$ & & & & & 1,952 \\
\hline & $(3,1)$ & 2 & 2 & $\begin{array}{c}\mathbb{Z}_{3}^{3} \\
0\end{array}$ & $\begin{array}{c}\mathbb{Z}_{3}^{3} \\
6\end{array}$ & & & & & 6 \\
\hline & $(4,1)$ & 2 & 1 & $\begin{array}{c}\mathbb{Z}_{3}^{4} \\
22 \\
\Delta(54)^{2} \\
88\end{array}$ & $\begin{array}{c}\mathbb{Z}_{3}^{4} \\
105\end{array}$ & & & & & 215 \\
\hline \multirow[t]{2}{*}{$\mathbb{Z}_{3} \times \mathbb{Z}_{6}$} & $(1,1)$ & 1 & 1 & $\begin{array}{c}\mathbb{Z}_{3}^{2} \times \mathbb{Z}_{6} \\
4,469\end{array}$ & & & & & & 4,493 \\
\hline & $(2,1)$ & 1 & 1 & $\begin{array}{c}\mathbb{Z}_{3}^{2} \times \mathbb{Z}_{6} \\
495\end{array}$ & & & & & & 540 \\
\hline \multirow[t]{4}{*}{$\mathbb{Z}_{4} \times \mathbb{Z}_{4}$} & $(1,1)$ & 3 & 3 & $\begin{array}{c}\left(D_{4}^{2} \times \mathbb{Z}_{4}^{2}\right) / \mathbb{Z}_{2} \\
2,258\end{array}$ & $\begin{array}{c}D_{4} \times \mathbb{Z}_{4}^{2} \times \mathbb{Z}_{2} \\
12,091\end{array}$ & $\begin{array}{c}\mathbb{Z}_{4}^{2} \times \mathbb{Z}_{2}^{3} \\
599\end{array}$ & & & & 14,953 \\
\hline & $(2,1)$ & 2 & 1 & $\begin{array}{c}D_{4} \times \mathbb{Z}_{4}^{2} \\
436 \\
\left(D_{4}^{2} \times \mathbb{Z}_{4}^{2}\right) / \mathbb{Z}_{2}^{2} \\
2,784\end{array}$ & $\begin{array}{c}D_{4} \times \mathbb{Z}_{4}^{2} \\
2,807\end{array}$ & & & & & 6,046 \\
\hline & $(3,1)$ & 2 & 2 & $\begin{array}{c}D_{4} \times \mathbb{Z}_{4}^{2} \\
875\end{array}$ & $\begin{array}{c}D_{4} \times \mathbb{Z}_{4}^{2} \\
2,039\end{array}$ & & & & & 2,920 \\
\hline & $(4,1)$ & 2 & 1 & $\begin{array}{c}\mathbb{Z}_{4}^{2} \times \mathbb{Z}_{2}^{2} \\
412 \\
\left(D_{4}^{2} \times \mathbb{Z}_{4}^{2}\right) / \mathbb{Z}_{2}^{2} \\
1,140\end{array}$ & $\begin{array}{c}\mathbb{Z}_{4}^{2} \times \mathbb{Z}_{2}^{2} \\
1,876\end{array}$ & & & & & 3,434 \\
\hline
\end{tabular}


[1] H. Ishimori, T. Kobayashi, H. Ohki, Y. Shimizu, H. Okada, and M. Tanimoto, Prog. Theor. Phys. Suppl. 183, 1 (2010).

[2] S. F. King and C. Luhn, Rep. Prog. Phys. 76, 056201 (2013).

[3] T. Kobayashi, H. P. Nilles, F. Plöger, S. Raby, and M. Ratz, Nucl. Phys. B768, 135 (2007).

[4] H. P. Nilles, M. Ratz, and P. K. Vaudrevange, Fortschr. Phys. 61, 493 (2013).

[5] T. Kobayashi, S. Raby, and R.-J. Zhang, Phys. Lett. B 593, 262 (2004).

[6] P. Ko, T. Kobayashi, J.-H. Park, and S. Raby, Phys. Rev. D 76, 035005 (2007).

[7] D. K. Mayorga Peña, H. P. Nilles, and P.-K. Oehlmann, J. High Energy Phys. 12 (2012) 024.

[8] B. Carballo-Pérez, E. Peinado, and S. Ramos-Sánchez, J. High Energy Phys. 12 (2016) 131.

[9] J. E. Kim, Phys. Rev. D 98, 055005 (2018).

[10] H. Abe, T. Kobayashi, H. Otsuka, Y. Takano, and T. H. Tatsuishi, Phys. Rev. D 94, 126020 (2016).

[11] T. Kobayashi, S. Nagamoto, S. Takada, S. Tamba, and T. H. Tatsuishi, Phys. Rev. D 97, 116002 (2018).

[12] F. Beye, T. Kobayashi, and S. Kuwakino, Phys. Lett. B 736, 433 (2014).

[13] H. Abe, K.-S. Choi, T. Kobayashi, and H. Ohki, Nucl. Phys. B820, 317 (2009).

[14] M. Berasaluce-González, P. G. Cámara, F. Marchesano, D. Regalado, and A. M. Uranga, J. High Energy Phys. 09 (2012) 059.

[15] F. Marchesano, D. Regalado, and L. Vazquez-Mercado, J. High Energy Phys. 09 (2013) 028.

[16] Y. Hamada, T. Kobayashi, and S. Uemura, J. High Energy Phys. 05 (2014) 116.

[17] L. J. Dixon, J. A. Harvey, C. Vafa, and E. Witten, Nucl. Phys. B261, 678 (1985).

[18] L. J. Dixon, J. A. Harvey, C. Vafa, and E. Witten, Nucl. Phys. B274, 285 (1986).

[19] D. Bailin and A. Love, Phys. Rep. 315, 285 (1999).

[20] S. Ramos-Sánchez, Fortschr. Phys. 57, 907 (2009).

[21] P. K. S. Vaudrevange, Grand unification in the heterotic brane world, Ph.D. thesis, Bonn University, 2008, arXiv:0812.3503.

[22] M. Fischer, M. Ratz, J. Torrado, and P. K. Vaudrevange, J. High Energy Phys. 01 (2013) 084.

[23] S. Ramos-Sánchez, J. Phys. Conf. Ser. 912, 012011 (2017).

[24] M. Blaszczyk, S. Groot-Nibbelink, O. Loukas, and S. Ramos-Sánchez, J. High Energy Phys. 10 (2014) 119.

[25] T. Kobayashi, S. Raby, and R.-J. Zhang, Nucl. Phys. B704, 3 (2005).

[26] W. Buchmüller, K. Hamaguchi, O. Lebedev, and M. Ratz, Phys. Rev. Lett. 96, 121602 (2006).

[27] J. E. Kim, J.-H. Kim, and B. Kyae, J. High Energy Phys. 06 (2007) 034.

[28] M. Blaszczyk, S. G. Nibbelink, M. Ratz, F. Ruehle, M. Trapletti, and P. K. S. Vaudrevange, Phys. Lett. B 683, 340 (2010).

[29] O. Lebedev and S. Ramos-Sánchez, Phys. Lett. B 684, 48 (2010).

[30] W. Buchmüller, K. Hamaguchi, O. Lebedev, S. RamosSánchez, and M. Ratz, Phys. Rev. Lett. 99, 021601 (2007).
[31] R. Kappl, H. P. Nilles, S. Ramos-Sánchez, M. Ratz, K. Schmidt-Hoberg, and P. K. S. Vaudrevange, Phys. Rev. Lett. 102, 121602 (2009).

[32] K.-S. Choi, H. P. Nilles, S. Ramos-Sánchez, and P. K. Vaudrevange, Phys. Lett. B 675, 381 (2009).

[33] F. Brümmer, R. Kappl, M. Ratz, and K. Schmidt-Hoberg, J. High Energy Phys. 04 (2010) 006.

[34] S. Krippendorf, H. P. Nilles, M. Ratz, and M. W. Winkler, Phys. Lett. B 712, 87 (2012).

[35] M. Badziak, S. Krippendorf, H. P. Nilles, and M. W. Winkler, J. High Energy Phys. 03 (2013) 094.

[36] J. E. Kim, J. High Energy Phys. 06 (2015) 114.

[37] H. P. Nilles, S. Ramos-Sánchez, P. K. S. Vaudrevange, and A. Wingerter, Comput. Phys. Commun. 183, 1363 (2012).

[38] T. Kobayashi, S. L. Parameswaran, S. Ramos-Sánchez, and I. Zavala, J. High Energy Phys. 05 (2012) 008.

[39] F. Plöger, S. Ramos-Sánchez, M. Ratz, and P. K. S. Vaudrevange, J. High Energy Phys. 04 (2007) 063.

[40] T. T. Burwick, R. K. Kaiser, and H. F. Müller, Nucl. Phys. B355, 689 (1991).

[41] J. Erler, D. Jungnickel, M. Spaliński, and S. Stieberger, Nucl. Phys. B397, 379 (1993).

[42] K.-S. Choi and T. Kobayashi, Nucl. Phys. B797, 295 (2008).

[43] L. J. Dixon, V. Kaplunovsky, and J. Louis, Nucl. Phys. B355, 649 (1991).

[44] S. Hamidi and C. Vafa, Nucl. Phys. B279, 465 (1987).

[45] L. J. Dixon, D. Friedan, E. J. Martinec, and S. H. Shenker, Nucl. Phys. B282, 13 (1987).

[46] J. A. Casas, F. Gómez, and C. Muñoz, Int. J. Mod. Phys. A 08, 455 (1993).

[47] T. Kobayashi and N. Ohtsubo, Int. J. Mod. Phys. A 09, 87 (1994).

[48] H. P. Nilles, S. Ramos-Sánchez, M. Ratz, and P. K. S. Vaudrevange, Phys. Lett. B 726, 876 (2013).

[49] N. G. Cabo Bizet, T. Kobayashi, D. K. Mayorga Peña, S. L. Parameswaran, M. Schmitz, and I. Zavala, J. High Energy Phys. 05 (2013) 076.

[50] S. Ramos-Sánchez, P. K. S. Vaudrevange, arXiv: 1811.00580.

[51] S. L. Parameswaran, S. Ramos-Sánchez, and I. Zavala, J. High Energy Phys. 01 (2011) 071.

[52] B. Dundee, S. Raby, and A. Westphal, Phys. Rev. D 82, 126002 (2010).

[53] S. Groot Nibbelink and P. K. S. Vaudrevange, J. High Energy Phys. 03 (2013) 142.

[54] M. Fischer, S. Ramos-Sánchez, and P. K. S. Vaudrevange, J. High Energy Phys. 07 (2013) 080.

[55] Y. Olguín-Trejo, R. Pérez-Martínez, and S. RamosSánchez, Tables of Abelian heterotic orbifolds and their flavor symmetries, http://cuerdas.fisica.unam.mx/ stringflavor/.

[56] H. P. Nilles and P. K. S. Vaudrevange, Adv. Ser. Dir. High Energy Phys. 22, 49 (2015).

[57] J. Lauer, J. Mas, and H. P. Nilles, Phys. Lett. B 226, 251 (1989).

[58] J. Lauer, J. Mas, and H. P. Nilles, Nucl. Phys. B351, 353 (1991).

[59] S. Ferrara, D. Lüst, and S. Theisen, Phys. Lett. B 233, 147 (1989). 
[60] D. Bailin, A. Love, W. A. Sabra, and S. Thomas, Mod. Phys. Lett. A 09, 1229 (1994).

[61] F. Feruglio, arXiv:1706.08749.

[62] J. T. Penedo and S. T. Petcov, arXiv:1806.11040.
[63] T. Kobayashi, N. Omoto, Y. Shimizu, K. Takagi, M. Tanimoto, and T. H. Tatsuishi, arXiv:1808.03012.

[64] T. Kobayashi, K. Tanaka, and T. H. Tatsuishi, Phys. Rev. D 98, 016004 (2018). 